\title{
Comparison of the Assumed Modes and Finite Element Models for Flexible Multi-Link Manipulators*
}

\author{
Rex J Theodore and Ashitava Ghosal \\ Department of Mechanical Engineering \\ Indian Institute of Science \\ Bangalore - 560 012, INDIA
}

\begin{abstract}
The objective of this paper is to compare discretization models namely the assumed modes and the finite elements to efficiently represent the link flexibility of robot manipulators. We present a systematic modeling procedure based on homogeneous transformation matrices, for spatial multi-link flexible manipulators with both revolute and prismatic joints. The Lagrangian formulation of dynamics, and computer algebra is employed to derive closed form equations of motion. We show that fewer number of mathematical operations are required for inertia matrix computation in case of finite element model compared to the assumed modes formulation, however since the number of state space equations are more, the numerical simulation time may be larger for finite element models. Use of finite element model to approximate flexibility usually gives rise to over-estimated stiffness matrix. We analytically show that over-estimation of structure stiffness may lead to unstable closed-loop response of the original manipulator system, using a model-based control law. We illustrate the complexity due to time-dependent frequency equation of assumed modes model arising in the case of a prismatic jointed flexible link with payload, and in case of manipulators having more than one link with revolute joints. We describe a novel method based on the differential form of the frequency equation to simulate such systems. A model-based decoupling control law is used to compare the dynamic responses of the manipulator system. The results are illustrated by numerical simulation for a flexible spatial RRP configuration robot.
\end{abstract}

${ }^{*}$ Updated version of the paper appeared in The International Journal of Robotics Research, Vol 14(2):91-111, 1995. 


\section{Introduction}

Initial studies of robotic systems have assumed rigid-body system models for the arm structures due to their rugged design and low operational speeds. However, as the demand on operational speed increases, and contemporary designs call for high payload capacity with relatively lightweight arm structures, a more detailed, accurate, and realistic system model is needed for the robot system design and for its control purposes. Hence in recent years, a great deal of research interest has been focussed on the modeling and control of lightweight flexible manipulators (Huston 1980, Sunada and Dubowsky 1981, Book 1984, Cannon and Schmitz 1984, Singh and Schy 1986 a, Baruh and Tadikonda 1989, Korolov and Chen 1989, Tosunoglu et al. 1992, De Luca and Siciliano 1993).

The overall structural flexibility in robotic manipulators, in general, is affected by both link and joint deflections. However, for a given robot design, their contribution to the system flexibility may vary considerably. The current industrial robots with their bulky and rugged link design tend to display relatively large joint deflections which seriously degrade the system precision (Good et al. 1985, Yang and Donath 1988). The space shuttle arm, on the other hand, displays dominant link compliance (Fresonke et al. 1988, Alberts et al. 1992). Although it is necessary to include both types of flexibilities in a general modeling procedure, in this paper we consider only the effect of link compliance.

\subsection{Review of Prior Work}

The robotic systems with flexible links are continuous dynamical systems characterized by infinite number of degrees of freedom, and are governed by nonlinear, coupled, ordinary and partial differential equations. The exact solution of such a mathematical model is normally not feasible, and the infinite dimensional model imposes severe constraint on the design of controllers as well (Book 1990, Shiffman 1993). Hence it is necessary that these continuous systems are discretized. Flexible links with rigid joints in robot manipulators are discretized by using assumed modes, finite elements, or lumped-parameters. The link deflections with respect to its rigid configuration, are usually assumed to be small and a linear theory of elasticity is selected. Researchers commonly utilize the Euler-Bernoulli beam theory (Timoshenko et al. 1974) to represent the dynamics of link flexibility, and neglect the effects of shear deformation and mass moment of inertia of a differential element along the length. For short(relative to its diameter), stubby beams, or for higher modes, the Timoshenko beam theory should be used which includes both these effects, but such 'stubby' links are likely to be essentially rigid (Bayo and Saharian 1986, Book 1990, Morris and Vidyasagar 1990).

In the assumed modes model formulation, the link flexibility is usually represented by the truncated finite modal series, in terms of spatial mode eigen functions and time-varying mode amplitudes. Although this method has been widely used in the literature (Book 1984, Hastings and Book 1986, Wang and Vidyasagar 1989, De Luca and Siciliano 1991, Li and 
Sankar 1993), there are several ways to choose link boundary conditions and mode eigen functions. For example, a clamped boundary condition leads to a physically measurable joint variable and simpler coefficients of the joint torques (Book 1984, Wang and Vidyasagar 1989). Pinned-pinned boundary conditions lead to ease in specifying the arm tip and have been used to advantage in trajectory control (Asada et al. 1990). Others have used free-free boundary conditions and described the link c.g. with rigid motion variables (Baruh and Tadikonda 1989). Hastings and Book(1986), and later Barbieri and Özgüner(1988) have reported that if the beam-to-hub inertia ratio is very small (in the order of 0.1 or less) the clamped condition yields better results compared to pinned boundary condition. They also reported experimental verifications. An interesting equivalence of these two cases is reported by Bellezza et al.(1990).

The finite element model on the other hand, has not gained much acceptance among researchers for use in the design of controllers for flexible-link robot manipulators (Book 1984, Oakley and Cannon 1988, Li and Sankar 1993), though it is generally considered as an useful tool especially in the design of robotic system itself (Sunada and Dubowsky 1981, Jonker 1990). In the finite element method, the boundary conditions and changes in geometry and physical properties can be accounted in a straightforward way. This advantage, in recent years, has been used to derive closed form equations of motion, and in the analysis of controllers (Usoro et al. 1986, Naganathan and Soni 1987, Jonker 1990, Chedmail et al. 1991). In this method, each flexible link is considered as an assemblage of finite number of elements, where every such element is a part of a continuous member of the link. By requiring that the displacements be compatible and the internal forces in balance at certain points called 'nodes', the entire link is compelled to act as one entity. The displacement at any point of the continuous element is expressed in terms of finite number of displacements at the nodal points multiplied by polynomial interpolation functions. The equations of motion for the overall robotic system is then derived by first deriving the equations of motion for a typical element and then suitably assembling the individual elements' equations of motion.

The lumped-parameter approach is best described by the so-called 'pseudo-joints'. This modeling technique assumes as many fictitious joints as necessary to appropriately describe the deflection of a flexible link. Each non-actuated fictitious joint is also accompanied by a linear spring to restrict the joint motion and represent the flexibility. If the vibrations of a link in different orthogonal planes are considered separately (two lateral and one longitudinal vibration) and each is represented by a pseudo-prismatic joint, then it can be said that this method models the first mode of vibration in each direction. Furthermore, three pseudo-revolute joints may be added to represent the effect of rotational vibrations. Huston(1980) adopted a similar approach utilizing the concept of 'quasi-coordinates'. Fresonke et al.(1988) developed an end-effector deflection prediction scheme in terms of the geometry dependent 'influence coefficients'. Tosunoglu et al.(1992) used this model for identification of inaccessible oscillations, in $n$-link flexible robotic systems. 


\subsection{Scope of Present Work}

The lumped-parameter model is rarely used in the design and analysis of flexible systems due to the difficulty in determining spring constants for the pseudo-joints. Several researchers (Book 1984, Cetinkunt and Book 1987, Asada et al. 1990, De Luca and Siciliano 1991, Cetinkunt and Ittoop 1992, Li and Sankar 1993) have used the assumed modes approach to derive closed form dynamic equations of motion for multi-link flexible manipulators. Others (Usoro et al. 1986, Naganathan and Soni 1987, Chedmail et al. 1991) have used finite element method for the same purpose. However, no attempt has been made to compare the two models used to represent the flexibility of links. Moreover, these works address mostly the revolute jointed flexible links, and the research on modeling prismatic jointed flexible manipulators is limited (Chalhoub and Ulsoy 1986, Yuh and Young 1991, Tadikonda and Baruh 1992).

In this work, we present a systematic modeling procedure for spatial multi-link flexible manipulators with both revolute and prismatic joints. In section 2, we describe discretization models namely assumed modes and finite elements to approximate the flexibility of EulerBernoulli type of links. We consider only the bending vibrations of manipulator links. The kinematics of multi-link flexible manipulators is derived using $4 \times 4$ homogeneous transformation matrices. We investigate the complexity due to time-dependent frequency equation of the assumed modes model in certain configuration of robots. The Lagrangian formulation of dynamics is employed and an algorithm is presented (in the appendix) to derive the closed form equations of motion more efficiently. In section 3, we present a model-based control law based on the nonlinear decoupling technique applied to the joint variables. In section 4 , a comparison of assumed modes and finite element models is presented. We show that only fewer number of mathematical operations are required for inertia matrix computation in case of finite element model compared to the assumed modes formulation. It is known that use of finite elements to approximate the flexibility, usually results in over estimated structure stiffness matrix for the system model (Meirovitch 1986). We show that over estimation of stiffness may lead to unstable closed-loop response of the original system using a model based control law. We also describe a novel method to account for the time-dependent frequency equation in dynamics computation of the assumed modes model. In section 5, we present numerical simulation results for a flexible, spatial RRP (Stanford Arm) configuration robot. The dynamic response of the arm due to model-based control law as presented in section 3, is compared for cases of assumed modes and finite element model formulations. In section 6 , we summarize the results and present some concluding remarks.

\section{$2 \quad$ Modeling}

The dynamics of rigid-arm manipulators is characterized by system of nonlinear, coupled, ordinary differential equations, but manipulators with flexible links being continuous (distributed) dynamical systems, are governed by nonlinear, coupled, ordinary and partial differ- 
ential equations. In this section, we describe the assumed modes, and finite element models to approximate the flexibility of links. We consider only the bending vibrations of flexible links ${ }^{1}$. We use Euler-Bernoulli beam theory (Timoshenko et al. 1974) to represent the dynamics of flexible links, and the kinematics of such manipulators, is represented utilizing $4 \times 4$ homogeneous transformation matrices. The computations then resulting from Lagrangian formulation of dynamics are used to derive the closed form equations of motion. These equations are free from assumptions of a nominal motion, and do not ignore the interaction of rigid and flexible generalized variables, though the link deflections with respect to its rigid configuration are assumed to be small.

\subsection{Flexible-Arm Kinematics}

By convention, the links of a flexible manipulator are numbered consecutively from 0 to $n$ starting from base of the manipulator to tip of the end-effector, where $n$ is the total number of links. We define the coordinate system $\left(X_{j}, Y_{j}, Z_{j}\right)$ on link $j$ with origin $O_{j}$ at the distal end (farthest from the base), oriented so that the $Z_{j}$ axis is along the axis of joint $j+1$. We also define the coordinate system $\left(\widehat{X}_{j}, \widehat{Y}_{j}, \widehat{Z}_{j}\right)$ on link $j$ with origin $\widehat{O}_{j}$ in such a way that when the link is in its undeformed configuration, the coordinate system $\left(X_{j}, Y_{j}, Z_{j}\right)$ is exactly coincident on the coordinate system $\left(\widehat{X}_{j}, \widehat{Y}_{j}, \widehat{Z}_{j}\right)$ (see figure 1 ).

The $4 \times 4$ homogeneous transformation matrix from coordinate system $\left(\widehat{X}_{j}, \widehat{Y}_{j}, \widehat{Z}_{j}\right)$ to coordinate system $\left(X_{j-1}, Y_{j-1}, Z_{j-1}\right)$ is given by,

$$
\mathbf{A}_{j}^{j-1}=\left(\begin{array}{cccc}
\cos \theta_{j} & -\sin \theta_{j} \cos \alpha_{j} & \sin \theta_{j} \sin \alpha_{j} & a_{j} \cos \theta_{j} \\
\sin \theta_{j} & \cos \theta_{j} \cos \alpha_{j} & -\cos \theta_{j} \sin \alpha_{j} & a_{j} \sin \theta_{j} \\
0 & \sin \alpha_{j} & \cos \alpha_{j} & d_{j} \\
0 & 0 & 0 & 1
\end{array}\right)
$$

where, $\theta_{j}, \alpha_{j}, d_{j}$, and $a_{j}$ are the Denavit-Hartenberg parameters representing relationship between coordinate systems $\left(\widehat{X}_{j}, \widehat{Y}_{j}, \widehat{Z}_{j}\right)$ and $\left(X_{j-1}, Y_{j-1}, Z_{j-1}\right)$ (Asada and Slotine 1986). Throughout this paper, $q_{r_{j}}$ denotes the joint variable: it is $\theta_{j}$ if joint $j$ is revolute, or $d_{j}$ if the joint is prismatic. Note that for the link with prismatic joint, $a_{j}=0$. The $4 \times 4$ homogeneous transformation matrix from coordinate system $\left(X_{j}, Y_{j}, Z_{j}\right)$ to $\left(\widehat{X}_{j}, \widehat{Y}_{j}, \widehat{Z}_{j}\right)$, caused by the deformation of link $j$-assuming small elastic deformations (Book 1984) is given by,

$$
\mathbf{E}_{j}^{j-1}=\left(\begin{array}{cccc}
1 & -\phi_{z_{j}} & \phi_{y_{j}} & \delta_{x_{j}} \\
\phi_{z_{j}} & 1 & -\phi_{x_{j}} & \delta_{y_{j}} \\
-\phi_{y_{j}} & \phi_{x_{j}} & 1 & \delta_{z_{j}} \\
0 & 0 & 0 & 1
\end{array}\right)
$$

\footnotetext{
${ }^{1}$ For most robotic manipulators in general, we can neglect the axial and torsional vibration components of the links because of their much greater rigidity in the axial direction and due to the structural design of robotic systems. However Sakawa and Luo(1989) reported that the bending vibrations are accompanied by torsional vibrations in certain classes of manipulators.
} 
where $\phi_{j}=\left(\phi_{x_{j}}, \phi_{y_{j}}, \phi_{z_{j}}\right)^{T}$ and $\delta_{j}=\left(\delta_{x_{j}}, \delta_{y_{j}}, \delta_{z_{j}}\right)^{T}$ describe the rotation and translation between the coordinate systems $\left(\widehat{X}_{j}, \widehat{Y}_{j}, \widehat{Z}_{j}\right)$ and $\left(X_{j}, Y_{j}, Z_{j}\right)$, respectively. Let $\widehat{\mathbf{T}}_{j}^{0}$ and $\mathbf{T}_{j}^{0}$ be the $4 \times 4$ homogeneous transformation matrices from coordinate systems $\left(\widehat{X}_{j}, \widehat{Y}_{j}, \widehat{Z}_{j}\right)$ and $\left(X_{j}, Y_{j}, Z_{j}\right)$ to the base coordinate system $\left(X_{0}, Y_{0}, Z_{0}\right)$, respectively, then

$$
\widehat{\mathbf{T}}_{j}^{0}=\left(\begin{array}{cc}
\widehat{\mathbf{R}}_{j}^{0} & \widehat{\mathbf{p}}_{j}^{0} \\
\mathbf{0} & 1
\end{array}\right)=\mathbf{A}_{1}^{0} \mathbf{E}_{1}^{0} \cdots \mathbf{A}_{j-1}^{j-2} \mathbf{E}_{j-1}^{j-2} \mathbf{A}_{j}^{j-1}
$$

and

$$
\mathbf{T}_{j}^{0}=\left(\begin{array}{cc}
\mathbf{R}_{j}^{0} & \mathbf{p}_{j}^{0} \\
\mathbf{0} & 1
\end{array}\right)=\mathbf{A}_{1}^{0} \mathbf{E}_{1}^{0} \cdots \mathbf{A}_{j-1}^{j-2} \mathbf{E}_{j-1}^{j-2} \mathbf{A}_{j}^{j-1} \mathbf{E}_{j}^{j-1}
$$

where $\widehat{\mathbf{R}}_{j}^{0}\left(\mathbf{R}_{j}^{0}\right)$ is the $3 \times 3$ rotation matrix, $\widehat{\mathbf{p}}_{j}^{0}\left(\mathbf{p}_{j}^{0}\right)$ is the $3 \times 1$ position vector, and $\mathbf{0}$ is the $1 \times 3$ zero vector.

The position vector of any point $(s)$ along the neutral axis of link $j$ can be expressed with respect to the base coordinate system $\left(X_{0}, Y_{0}, Z_{0}\right)$ as,

$$
\mathbf{r}_{j}^{0}=\mathbf{p}_{j-1}^{0}+\widehat{\mathbf{R}}_{j}^{0} \mathbf{r}_{j}
$$

where,

$$
\mathbf{r}_{j}=\left\{\begin{array}{l}
\left(\begin{array}{l}
s \\
0 \\
0
\end{array}\right)+\left(\begin{array}{c}
0 \\
v_{j}(s, t) \\
w_{j}(s, t)
\end{array}\right) \text { if joint } j \text { is revolute } \\
\left(\begin{array}{l}
0 \\
0 \\
s
\end{array}\right)+\left(\begin{array}{c}
u_{j}(s, t) \\
v_{j}(s, t) \\
0
\end{array}\right) \text { if joint } j \text { is prismatic }
\end{array}\right.
$$

and $u_{j}(s, t), v_{j}(s, t), w_{j}(s, t)$ are displacements with reference to the neutral axis of flexible link $j$ at a distance $s$ and at time $t$, due to flexibility in the respective directions. Note that the dependence of $u_{j}, v_{j}, w_{j}$ on the spatial coordinate $(s)$, makes the system infinite dimensional, leading to coupled ordinary and partial differential equations of motion.

\subsubsection{Assumed Modes Model}

In the assumed modes model, the flexible displacements: $u_{j}(s, t), v_{j}(s, t), w_{j}(s, t)$ are usually described by a truncated modal series, in terms of spatial mode eigen functions and time dependent mode amplitudes, in each direction as,

$$
\begin{aligned}
u_{j}(\eta, t) & =\sum_{i=1}^{n_{j}} \psi_{i}^{u_{j}}(\eta) \xi_{i}^{u_{j}}(t) \\
v_{j}(\eta, t) & =\sum_{i=1}^{n_{j}} \psi_{i}^{v_{j}}(\eta) \xi_{i}^{v_{j}}(t) \\
w_{j}(\eta, t) & =\sum_{i=1}^{n_{j}} \psi_{i}^{w_{j}}(\eta) \xi_{i}^{w_{j}}(t)
\end{aligned}
$$


where $\eta=\frac{s}{l_{j}}, l_{j}$ is the length of flexible link $j$, and $n_{j}$ is the number of modes used to describe the deflection of link $j$. It should be mentioned that for the flexible link with a revolute joint, $l_{j} \equiv a_{j}$, and for a flexible link with prismatic joint, $l_{j} \equiv d_{j}$. The $4 \times 4$ homogeneous transformation matrix, $\mathbf{E}_{j}^{j-1}$ for link $j$, is given by,

if joint $j$ is revolute:

$$
\mathbf{E}_{j}^{j-1}=\mathbf{I}+\sum_{i=1}^{n_{j}}\left(\begin{array}{cccc}
0 & -\frac{\partial \psi_{i}^{v_{j}}(1)}{\partial \eta} \xi_{i}^{v_{j}}(t) & \frac{\partial \psi_{i}^{w_{j}}(1)}{\partial \eta} \xi_{i}^{w_{j}}(t) & 0 \\
\frac{\partial \psi_{i}^{v_{j}}(1)}{\partial \eta} \xi_{i}^{v_{j}}(t) & 0 & 0 & \psi_{i}^{v_{j}}(1) \xi_{i}^{v_{j}}(t) \\
-\frac{\partial \psi_{i}^{w_{j}}(1)}{\partial \eta} \xi_{i}^{w_{j}}(t) & 0 & 0 & \psi_{i}^{w_{j}}(1) \xi_{i}^{w_{j}}(t) \\
0 & 0 & 0 & 0
\end{array}\right)
$$

if joint $j$ is prismatic:

$$
\mathbf{E}_{j}^{j-1}=\mathbf{I}+\sum_{i=1}^{n_{j}}\left(\begin{array}{cccc}
0 & 0 & \frac{\partial \psi_{i}^{u_{j}}(1)}{\partial \eta} \xi_{i}^{u_{j}}(t) & \psi_{i}^{u_{j}}(1) \xi_{i}^{u_{j}}(t) \\
0 & 0 & -\frac{\partial \psi_{i}^{v_{j}}(1)}{\partial \eta} \xi_{i}^{v_{j}}(t) & \psi_{i}^{v_{j}}(1) \xi_{i}^{v_{j}}(t) \\
-\frac{\partial \psi_{i}^{u_{j}}(1)}{\partial \eta} \xi_{i}^{u_{j}}(t) & \frac{\partial \psi_{i}^{v_{j}}(1)}{\partial \eta} \xi_{i}^{v_{j}}(t) & 0 & 0 \\
0 & 0 & 0 & 0
\end{array}\right)
$$

where $\mathbf{I}$ is the $4 \times 4$ identity matrix and note that all variables in the matrix are evaluated at $\eta=1$, tip of the link $j$. The generalized flexible deformation variables in this case is therefore, $\mathbf{q}_{f_{j}}=\left(\xi_{1}^{u_{j}}(t), \xi_{1}^{v_{j}}(t), \xi_{1}^{w_{j}}(t), \cdots, \xi_{n_{j}}^{u_{j}}(t), \xi_{n_{j}}^{v_{j}}(t), \xi_{n_{j}}^{w_{j}}(t)\right)^{T}$.

The selection of 'appropriate' or 'best' mode eigen functions for a given flexible manipulator system is not a clearly answered problem (Book 1990, Bellezza et al. 1990). We choose "clamped-mass" mode eigen functions for flexible links of the manipulator system. For flexible link manipulator systems, it is reasonable to suppose that the inertia of a lightweight link is small compared to the hub inertia (De Luca and Siciliano 1991), and indeed it is reported that for links with small beam-to-hub inertia ratio, 'clamped' boundary conditions approximate the controlled end of the flexible link quite accurately (Hastings and Book 1986, Barbieri and Özgüner 1988). Concerning the boundary condition of distal end of the flexible link, it is usually assumed that the link end is 'free' of dynamic constraints (Singh and Schy 1986 a, Wang and Vidyasagar 1989, Yuh and Young 1991, Tadikonda and Baruh 1992), however, it is more correct to consider 'mass' boundary conditions representing balance of moment and shearing force due to other links of the serial structure of robot manipulators 
and the payload (Low 1989, Oakley and Cannon 1989, Book 1990, De Luca and Siciliano 1991). Thus the mode eigen function $\psi(\eta)$ satisfying clamped-mass boundary conditions with Euler-Bernoulli beam theory for bending vibrations is given by,

$$
\psi_{i}(\eta)=C_{i}\left[\cos \left(\beta_{i} \eta\right)-\cosh \left(\beta_{i} \eta\right)+\nu_{i}\left(\sin \left(\beta_{i} \eta\right)-\sinh \left(\beta_{i} \eta\right)\right)\right]
$$

where,

$$
\nu_{i}=\frac{\sin \beta_{i}-\sinh \beta_{i}+M \beta_{i}\left(\cos \beta_{i}-\cosh \beta_{i}\right)-D \beta_{i}{ }^{2}\left(\sin \beta_{i}+\sinh \beta_{i}\right)}{\cos \beta_{i}+\cosh \beta_{i}-M \beta_{i}\left(\sin \beta_{i}-\sinh \beta_{i}\right)-D \beta_{i}{ }^{2}\left(\cos \beta_{i}-\cosh \beta_{i}\right)}
$$

and $\beta_{i}$ are solutions of the frequency equation,

$$
\begin{gathered}
\left(1+\cosh \beta_{i} \cos \beta_{i}\right)-M \beta_{i}\left(\cosh \beta_{i} \sin \beta_{i}-\sinh \beta_{i} \cos \beta_{i}\right) \\
-J \beta_{i}{ }^{3}\left(\cosh \beta_{i} \sin \beta_{i}+\sinh \beta_{i} \cos \beta_{i}\right)+M J \beta_{i}{ }^{4}\left(1-\cosh \beta_{i} \cos \beta_{i}\right) \\
-D^{2} \beta_{i}{ }^{4}\left(1-\cosh \beta_{i} \cos \beta_{i}\right)-2 D \beta_{i}{ }^{2} \sinh \beta_{i} \sin \beta_{i}=0
\end{gathered}
$$

where $M=\frac{M_{L}}{\rho A l}, \quad J=\frac{J_{L}}{\rho A l^{3}}$, and $D=\frac{M_{D L}}{\rho A l^{2}}$. Note that $\rho, A, l$ are density of the material, area of cross section, and length of the flexible link $j$ respectively. $M_{L}$ is the constant sum of all masses beyond the flexible link $j$, and $J_{L}$ is the moment of inertia due to other links and payload "seen" at distal end of the flexible link $j . M_{D L}$ accounts for the contributions of masses of distal links, i.e., non-collocated at the end of link $j$, weighted by the relative distance from shearing axis at the end of flexible link $j$ (De Luca and Siciliano 1991). The $C_{i}$ are constants which normalizes the mode eigen functions so that, $\int_{0}^{1}\left[\psi_{i}(\eta)\right]^{2} d \eta=1$.

Equation(12) can be readily solved for flexible manipulators with one link having a revolute joint, but poses a problem for a prismatic jointed flexible link with payload or with an 'augmented body' (for example a wrist), and for flexible manipulators having more than one link with revolute joints. In case of prismatic joint with a payload, the length of the translating flexible beam $(l)$ is a function of time ${ }^{2}$. In the case of flexible manipulators having more than one link with revolute joints, $M_{D L}$ becomes a function of the configuration of successive links (De Luca and Siciliano 1991), and for flexible manipulators having more than two links with revolute joints, in addition to $M_{D L}$, the moment of inertia $\left(J_{L}\right)$ due to other links of serial nature and payload "seen" at distal end of the first link will be a function of time. For example, for a planar 3R flexible robot, the rotary inertia due to last two links, "seen" at distal end of the first link is given by, $J_{L}=I_{2}+I_{3}+m_{2} l_{2}^{2}+m_{3}\left(l_{2}^{2}+l_{3}^{2}+2 l_{2} l_{3} \cos \theta_{3}\right)$ where $m, l$, and $I$ are mass and length of the links, and the joint inertias respectively. Note that the joint variable $\theta_{3}$ is a function of time. Although time dependency of the frequency equation was pointed out by De Luca and Siciliano(1991) for revolute jointed planar flexible multi-link manipulators, and by Tadikonda and Baruh(1992) for the problem of a translating

\footnotetext{
${ }^{2}$ For a prismatic joint, we must ensure that the length $l$ satisfies Euler-Bernoulli beam assumptions viz., length of the beam $l$ must always be much greater than the beam cross sectional dimensions (Timoshenko et al. 1974, Chalhoub and Ulsoy 1986).
} 
flexible beam deploying a payload from a fixed base, they have not used the exact mode eigen functions of 'clamped-mass' conditions, because of the considerable increase in complexity of model derivation.

In cases where the clamped-mass frequency equation becomes time dependent, the solutions $\left(\beta_{i}\right)$ have to be computed by either using a root finding algorithm at each time step of integration, or by using a "table look-up" approach (Low 1989). The former approach may lead to considerable increase in computational time, while the latter requires a large storage space for the specified accuracy. In section 4, we present a novel method to solve the time-dependent frequency equation together with the dynamic equations of motion.

\subsubsection{Finite Element Model}

In this method, each flexible link is considered as an assemblage of finite number of elements, where every such element is a part of a continuous member of the link. By requiring that the displacements be compatible and the internal forces in balance at certain points called 'nodes', the entire link is compelled to act as one entity. The displacement at any point of the continuous element is then expressed in terms of finite number of displacements at the nodal points multiplied by polynomial interpolation functions. Let PQ be one such element $i$ on link $j$, with nodes $i$ and $i+1$ as shown in figure 2 . Then the local position vector $\mathbf{r}_{j i}$ of element $i$ is given as,

$$
\mathbf{r}_{j i}=\left\{\begin{array}{l}
\left(\begin{array}{c}
(i-1) l_{j i}+s \\
0 \\
0
\end{array}\right)+\left(\begin{array}{c}
0 \\
v_{j i}(s, t) \\
w_{j i}(s, t)
\end{array}\right) \text { if joint } j \text { is revolute } \\
\left(\begin{array}{c}
0 \\
0 \\
(i-1) l_{j i}+s
\end{array}\right)+\left(\begin{array}{c}
u_{j i}(s, t) \\
v_{j i}(s, t) \\
0
\end{array}\right) \text { if joint } j \text { is prismatic }
\end{array}\right.
$$

where $l_{j i}$ is the length of element $i$, and

if joint $j$ is revolute:

$$
\begin{aligned}
v_{j i}(s, t) & =H_{0 i}^{1}(s) \delta_{i}^{v_{j}}(t)+H_{1 i}^{1}(s) \phi_{i}^{w_{j}}(t)+H_{0(i+1)}^{1}(s) \delta_{i+1}^{v_{j}}(t)+H_{1(i+1)}^{1}(s) \phi_{i+1}^{w_{j}}(t) \\
w_{j i}(s, t) & =H_{0 i}^{1}(s) \delta_{i}^{w_{j}}(t)+H_{1 i}^{1}(s) \phi_{i}^{v_{j}}(t)+H_{0(i+1)}^{1}(s) \delta_{i+1}^{w_{j}}(t)+H_{1(i+1)}^{1}(s) \phi_{i+1}^{v_{j}}(t)(14)
\end{aligned}
$$

if joint $j$ is prismatic:

$$
\begin{aligned}
& u_{j i}(s, t)=H_{0 i}^{1}(s) \delta_{i}^{u_{j}}(t)+H_{1 i}^{1}(s) \phi_{i}^{v_{j}}(t)+H_{0(i+1)}^{1}(s) \delta_{i+1}^{u_{j}}(t)+H_{1(i+1)}^{1}(s) \phi_{i+1}^{v_{j}}(t) \\
& v_{j i}(s, t)=H_{0 i}^{1}(s) \delta_{i}^{v_{j}}(t)+H_{1 i}^{1}(s) \phi_{i}^{u_{j}}(t)+H_{0(i+1)}^{1}(s) \delta_{i+1}^{v_{j}}(t)+H_{1(i+1)}^{1}(s) \phi_{i+1}^{u_{j}}(t)(15)
\end{aligned}
$$

The $\delta_{j i}=\left(\delta_{i}^{u_{j}}(t), \delta_{i}^{v_{j}}(t), \delta_{i}^{w_{j}}(t)\right)^{T}$, and $\phi_{j i}=\left(\phi_{i}^{u_{j}}(t), \phi_{i}^{v_{j}}(t), \phi_{i}^{w_{j}}(t)\right)^{T}$ denote the transverse flexural displacements and the flexural rotations of node $i$, respectively in the respective 
directions. It should be noted that for the flexible link with a revolute joint, $l_{j i} \equiv\left(a_{j} / n_{j}\right)$, and for a flexible link with prismatic joint, $l_{j i} \equiv\left(d_{j} / n_{j}\right)$, where $n_{j}$ is the number of finite elements in the link $j$. The interpolation functions are given as (Meirovitch 1986):

$$
\begin{aligned}
& H_{0 i}^{1}(s)=1-3\left(\frac{s}{l_{j i}}\right)^{2}+2\left(\frac{s}{l_{j i}}\right)^{3} \\
& H_{1 i}^{1}(s)=s\left(\frac{s}{l_{j i}}-1\right)^{2} \\
& H_{0(i+1)}^{1}(s)=\left(\frac{s}{l_{j i}}\right)^{2}\left(3-2 \frac{s}{l_{j i}}\right) \\
& H_{1(i+1)}^{1}(s)=\frac{s^{2}}{l_{j i}}\left(\frac{s}{l_{j i}}-1\right)
\end{aligned}
$$

In this case, the $4 \times 4$ homogeneous transformation matrix $\mathbf{E}_{j}^{j-1}$ due to the deformations of link $j$ is given by,

if joint $j$ is revolute:

$$
\mathbf{E}_{j}^{j-1}=\left(\begin{array}{cccc}
1 & -\phi_{n_{j}+1}^{w_{j}} & \phi_{n_{j}+1}^{v_{j}} & 0 \\
\phi_{n_{j}+1}^{w_{j}} & 1 & 0 & \delta_{n_{j}+1}^{v_{j}} \\
-\phi_{n_{j}+1}^{v_{j}} & 0 & 1 & \delta_{n_{j}+1}^{w_{j}} \\
0 & 0 & 0 & 1
\end{array}\right)
$$

if joint $j$ is prismatic:

$$
\mathbf{E}_{j}^{j-1}=\left(\begin{array}{cccc}
1 & 0 & \phi_{n_{j}+1}^{v_{j}} & \delta_{n_{j}+1}^{u_{j}} \\
0 & 1 & -\phi_{n_{j}+1}^{u_{j}} & \delta_{n_{j}+1}^{v_{j}} \\
-\phi_{n_{j}+1}^{v_{j}} & \phi_{n_{j}+1}^{u_{j}} & 1 & 0 \\
0 & 0 & 0 & 1
\end{array}\right)
$$

and $\mathbf{q}_{f_{j}}=\left(\delta_{j 1}^{T}(t), \phi_{j 1}^{T}(t), \cdots, \delta_{j\left(n_{j}+1\right)}^{T}(t), \phi_{j\left(n_{j}+1\right)}^{T}(t)\right)^{T}$ denotes the generalized flexible deformation variables.

\subsection{Dynamic Equations of Motion}

The dynamic equations of motion are obtained using the Lagrange's formulation of dynamics. It may be noted that the generalized force corresponding to joint variable $q_{r_{j}}$ is the joint input $\Gamma_{j}$ (torque $\tau_{j}$ for a revolute joint, or force $F_{j}$ for a prismatic joint). For the flexible deformation variables $\left(\mathbf{q}_{f}\right)$ the corresponding generalized force will be "zero", if the corresponding elastic deflections or rotations have no displacement at those locations where external forces 
are applied, and note that this corresponds to the case when 'clamped' condition is used for controlled end of the link (Book 1984, Wang and Vidyasagar 1989). It should be noted that other conditions for controlled end of the link, such as 'pinned' condition (Asada et al. 1990), or 'free' condition (Baruh and Tadikonda 1989) will have "non-zero" generalized forces corresponding to the generalized flexible deformation variables.

The general form of Lagrange's equations (for clamped condition) are then,

for joint variable $q_{r_{j}}$ :

$$
\frac{d}{d t}\left(\frac{\partial T}{\partial \dot{q}_{r_{j}}}\right)-\frac{\partial T}{\partial q_{r_{j}}}+\frac{\partial V}{\partial q_{r_{j}}}=\Gamma_{j}
$$

for flexible deformation variable $q_{f_{j i}}$ :

$$
\frac{d}{d t}\left(\frac{\partial T}{\partial \dot{q}_{f_{j i}}}\right)-\frac{\partial T}{\partial q_{f_{j i}}}+\frac{\partial V}{\partial q_{f_{j i}}}=0
$$

where, $T$ is the total kinetic energy of the flexible manipulator system, and $V$ is the total potential energy due to elastic deformations and gravity.

\subsubsection{Kinetic Energy}

The total kinetic energy of flexible-link manipulator system is due to the motions of joints and links, and kinetic energy due to the payload. The kinetic energy for a revolute joint $j$, if considered as mass with rotary inertia about the axis of revolution is given by,

$$
T_{\text {joint }_{j}}=\frac{1}{2} \Omega_{j}^{0^{T}} \mathbf{I}_{j} \Omega_{j}^{0}+\frac{1}{2} m_{j}\left(\frac{d \mathbf{p}_{j-1}^{0}}{d t}\right)^{T}\left(\frac{d \mathbf{p}_{j-1}^{0}}{d t}\right)
$$

where $m_{j}$ is the mass of the joint hub $j, \mathbf{p}_{j-1}^{0}$ is position vector of the joint $j, \mathbf{I}_{j}$, and $\Omega_{j}^{0}$ are the joint inertia matrix, and the angular velocity vector of joint $j$, respectively. In the case of prismatic joint $j$, at any instant of time, a part of the translating beam is outside the joint hub and is free to vibrate, while the remaining part of the beam is inside the joint hub and is restrained from vibrating. The kinetic energy due to part of the beam inside the joint hub is given by,

$$
T_{\text {joint }_{j}}=\frac{1}{2} \int_{\left(l_{j}-l_{j}^{0}\right)}^{0} \rho_{j} A_{j}\left(\frac{d \widehat{\mathbf{r}}_{j}^{0}}{d t}\right)^{T}\left(\frac{d \widehat{\mathbf{r}}_{j}^{0}}{d t}\right) d s
$$

where

$$
\widehat{\mathbf{r}}_{j}^{0}=\mathbf{p}_{j-1}^{0}+s \widehat{\mathbf{z}}_{j}^{0}
$$

and $\widehat{\mathbf{z}}_{j}^{0}$ is the third column vector of the rotation matrix $\widehat{\mathbf{R}}_{j}^{0}$, and $l_{j}^{0}, l_{j}$ are the total length of the translating beam, and length of the beam outside the joint hub at time $t$, respectively. 
The kinetic energy of a differential mass, $d m$, on flexible link $j$ of the manipulator can be expressed as,

$$
d T_{\text {link }_{j}}=\frac{1}{2} d m\left(\frac{d \mathbf{r}_{j}^{0}}{d t}\right)^{T}\left(\frac{d \mathbf{r}_{j}^{0}}{d t}\right)
$$

Under the assumption that the links are slender beams (Timoshenko et al. 1974), the kinetic energy of the flexible link $j$ can be obtained by integrating equation(23) over the length $\left(l_{j}\right)$ of the link as,

$$
T_{l i n k_{j}}=\frac{1}{2} \int_{0}^{l_{j}} \rho_{j} A_{j}\left(\frac{d \mathbf{r}_{j}^{0}}{d t}\right)^{T}\left(\frac{d \mathbf{r}_{j}^{0}}{d t}\right) d s
$$

where $\rho_{j}$ is the density of the material, $A_{j}$ is the cross-sectional area of the link.

The kinetic energy due to the payload is given by,

$$
T_{\text {payload }}=\frac{1}{2} m_{p}\left(\frac{d \mathbf{p}_{n}^{0}}{d t}\right)^{T}\left(\frac{d \mathbf{p}_{n}^{0}}{d t}\right)
$$

where $\mathbf{p}_{n}^{0}$ is position vector of tip of the end-effector(see equation(4)), and $m_{p}$ is mass of the payload.

\subsubsection{Potential Energy}

The potential energy of the flexible manipulator system arises from two sources - due to the deformation of links and due to gravity. Assuming slender beam type of links and neglecting the axial and torsional vibration of links, the potential energy due to bending deformations (Timoshenko et al. 1974) of link $j$ about the transverse $\widehat{Y}_{j}$ and $\widehat{Z}_{j}$ axes, is given by,

$$
V_{f j}=\frac{1}{2} \int_{0}^{l_{j}}\left(E_{j} I_{j y}\left(\frac{\partial^{2} v_{j}(s, t)}{\partial s^{2}}\right)^{2}+E_{j} I_{j z}\left(\frac{\partial^{2} w_{j}(s, t)}{\partial s^{2}}\right)^{2}\right) d s
$$

where $E_{j}$ is the Young's modulus, $I_{j y}, I_{j z}$ are the area moments of inertia about respective axes, of link $j$. Note that for flexible link $j$ with prismatic joint, the bending deformations of the link in the transverse $\widehat{X}_{j}$ and $\widehat{Y}_{j}$ axes have to be considered as opposed to the above equation.

The gravitational potential energy due to the mass of joint hub and due to the elastic link $j$ is of the form,

$$
V_{g j}=m_{j} \mathbf{g}^{T} \mathbf{p}_{j-1}^{0}+\int_{0}^{l_{j}} \rho_{j} A_{j} \mathbf{g}^{T} \mathbf{r}_{j}^{0} d s
$$

where $\mathbf{g}$ is the gravity vector in the inertial coordinate system $\{0\}$. The gravitational potential energy due to the payload mass is given by,

$$
V_{g_{\text {payload }}}=m_{p} \mathbf{g}^{T} \mathbf{p}_{n}^{0}
$$

The system's total potential energy $(V)$ is then, sum of potential energies (equations(26-27)) over all the links, and due to the payload(equation(28)). 
Remark: It should be noted that in case of finite element model, the kinetic $\left(T_{j i}\right)$, and potential $\left(V_{j i}\right)$ energies are obtained first for each element $i$ of link $j$ and then summing over all the elements $\left(n_{j}\right)$, we obtain the kinetic $\left(T_{j}\right)$, and potential $\left(V_{j}\right)$ energies for the link $j$.

\subsubsection{Closed form Equations of Motion}

Symbolic closed form equations are shown to be most efficient to implement real-time control algorithms for manipulator dynamical systems (Ju and Mansour 1989, De Luca and Siciliano 1991, Cetinkunt and Ittoop 1992). The closed form dynamic equations of motion for flexible link manipulators can be derived using symbolic manipulation software such as REDUCE(Rayna 1987) or MACSYMA(Annon 1987). The resulting equations of motion (see appendix for the algorithm) in the matrix form can be written as,

$$
\left(\begin{array}{ll}
\mathbf{M}_{r r} & \mathbf{M}_{r f} \\
\mathbf{M}_{r f}^{T} & \mathbf{M}_{f f}
\end{array}\right)\left(\begin{array}{c}
\ddot{\mathbf{q}}_{r} \\
\ddot{\mathbf{q}}_{f}
\end{array}\right)+\left(\begin{array}{c}
\mathbf{h}_{r}(\mathbf{q}, \dot{\mathbf{q}}) \\
\mathbf{h}_{f}(\mathbf{q}, \dot{\mathbf{q}})
\end{array}\right)+\left(\begin{array}{c}
\mathbf{c}_{r}(\mathbf{q}) \\
\mathbf{c}_{f}(\mathbf{q})
\end{array}\right)+\left(\begin{array}{cc}
\mathbf{0} & \mathbf{0} \\
\mathbf{0} & \mathbf{K}
\end{array}\right)\left(\begin{array}{c}
\mathbf{q}_{r} \\
\mathbf{q}_{f}
\end{array}\right)=\left(\begin{array}{c}
\Gamma \\
\mathbf{0}
\end{array}\right)
$$

where $\mathbf{q}=\left(\mathbf{q}_{r}^{T}, \mathbf{q}_{f}^{T}\right)^{T}$, is the $n$-vector of generalized joint $\left(\mathbf{q}_{r}\right)$, and $N$-vector of flexible deformation $\left(\mathbf{q}_{f}\right)$ variables, $\mathbf{M}$ is the $(n+N) \times(n+N)$ configuration dependent generalized mass matrix, $\mathbf{h}$ is the $(n+N)$-vector of Coriolis, and centrifugal terms and the terms accounting for the interaction of joint variables and their rates with flexible variables and their rates, c is the $(n+N)$-vector of gravitational terms, $\mathbf{K}$ is the $N \times N$ flexural structure stiffness matrix of the system, $\Gamma$ is the $n$-vector of input torques (or forces) applied at the joints, and $\mathbf{0}$ is the zero matrix/vector with appropriate dimensions.

\section{Model-based Control Law}

The control objective of flexible manipulators is mainly to stabilize the vibrations that are excited during motion of manipulator arms and damping them out as fast as possible at the end of the motion. To this end, the solutions suggested in the literature range from passive damping methods (Alberts et al. 1992) to active damping methods. Some of the methods for active control of flexible vibrations are based on: linear control system design methods such as PD compensators (Oakley and Cannon 1988), and optimal controllers(LQR) (Cannon and Schmitz 1984, Chiang et al. 1991), transfer function approach (Wang and Vidyasagar 1989), inverse dynamics techniques (Singh and Schy 1986 a, Asada et al. 1990), robust control design (Korolov and Chen 1989), nonlinear decoupling methods (Singh and Schy 1986 b, Das and Singh 1990, Chedmail et al. 1991, De Luca and Siciliano 1993), singular perturbation methods where the elastic and the rigid motions are assumed to be time separable (Siciliano and Book 1988), and adaptive control methods (Rovner and Cannon 1987). In this section, we present a control law design based on nonlinear decoupling method applied to the joint variables. This model-based decoupling control law, while tracking the 
desired joint trajectories during the gross motion of robot arms, does not drive the flexible variables to zero. A second control loop derived using the LQR method and based on linearized model of the closed-loop equations of motion around the final configuration of the arm (Singh and Schy 1986 b), performs an active damping of the flexible vibrations at the end of gross motion of links.

The open-loop equations of motion for the manipulator system, as developed in the previous section, can be written as

$$
\begin{array}{r}
\mathbf{M}_{r r} \ddot{\mathbf{q}}_{r}+\mathbf{M}_{r f} \ddot{\mathbf{q}}_{f}+\mathbf{h}_{r}(\mathbf{q}, \dot{\mathbf{q}})+\mathbf{c}_{r}(\mathbf{q})=\Gamma \\
\mathbf{M}_{r f}^{T} \ddot{\mathbf{q}}_{r}+\mathbf{M}_{f f} \ddot{\mathbf{q}}_{f}+\mathbf{h}_{f}(\mathbf{q}, \dot{\mathbf{q}})+\mathbf{c}_{f}(\mathbf{q})+\mathbf{K} \mathbf{q}_{f}=\mathbf{0}
\end{array}
$$

A model-based control, analogous to the one for rigid manipulators, can be written for multi-link flexible manipulator system if we consider the effects of flexible vibration as a deterministic disturbance on the nominal rigid body motion. We proceed as follows:

First solve for $\ddot{\mathbf{q}}_{f}$ from equation(31) as

$$
\ddot{\mathbf{q}}_{f}=-\mathbf{M}_{f f}^{-1}\left(\mathbf{M}_{r f}^{T} \ddot{\mathbf{q}}_{r}+\mathbf{h}_{f}+\mathbf{c}_{f}+\mathbf{K} \mathbf{q}_{f}\right)
$$

then substitute $\ddot{\mathbf{q}}_{f}$ in equation(30), and finally rearrange to get

$$
\left(\mathbf{M}_{r r}-\mathbf{M}_{r f} \mathbf{M}_{f f}^{-1} \mathbf{M}_{r f}^{T}\right) \ddot{\mathbf{q}}_{r}+\left(\mathbf{h}_{r}+\mathbf{c}_{r}-\mathbf{M}_{r f} \mathbf{M}_{f f}^{-1}\left(\mathbf{h}_{f}+\mathbf{c}_{f}+\mathbf{K} \mathbf{q}_{f}\right)\right)=\Gamma
$$

Equation(33) is similar in form to that of a rigid manipulator with $\left(\mathbf{M}_{r r}-\mathbf{M}_{r f} \mathbf{M}_{f f}^{-1} \mathbf{M}_{r f}^{T}\right)$ as an equivalent mass matrix, and $\left(\mathbf{h}_{r}+\mathbf{c}_{r}-\mathbf{M}_{r f} \mathbf{M}_{f f}^{-1}\left(\mathbf{h}_{f}+\mathbf{c}_{f}+\mathbf{K} \mathbf{q}_{f}\right)\right)$ as an equivalent nonlinear term. We note that the control vector $\Gamma$ is of the same rank as the rigid-body degrees of freedom (or the number of joints), and the matrix $\left(\mathbf{M}_{r r}-\mathbf{M}_{r f} \mathbf{M}_{f f}^{-1} \mathbf{M}_{r f}^{T}\right)$ is symmetric and positive definite (see appendix).

Following nonlinear decoupling theory, we can write a model-based controller, so that the coupled, nonlinear flexible manipulator system is reduced to a set of double integrators corresponding to the joint variables $\left(\mathbf{q}_{r}\right)$, and a set of coupled, nonlinear dynamic equations corresponding to the generalized flexible variables $\left(\mathbf{q}_{f}\right)$. This model-based controller is then given as,

$$
\boldsymbol{\Gamma}=\left(\mathbf{M}_{r r}-\mathbf{M}_{r f} \mathbf{M}_{f f}^{-1} \mathbf{M}_{r f}^{T}\right) \mathbf{u}_{I}+\left(\mathbf{h}_{r}+\mathbf{c}_{r}-\mathbf{M}_{r f} \mathbf{M}_{f f}^{-1}\left(\mathbf{h}_{f}+\mathbf{c}_{f}+\mathbf{K} \mathbf{q}_{f}\right)\right)
$$

where $\mathbf{u}_{I}$ is the new control input and can be chosen for a specified joint trajectory as

$$
\mathbf{u}_{I}=-\mathbf{G}_{p} \mathbf{q}_{r}-\mathbf{G}_{v} \dot{\mathbf{q}}_{r}+\mathbf{r}(t)
$$

where $\mathbf{G}_{p}$ and $\mathbf{G}_{v}$ are constant diagonal position and velocity gain matrices for the joint variables, respectively. The reference input vector, $\mathbf{r}(t)$, can be chosen as,

$$
\mathbf{r}(t)=\ddot{\mathbf{q}}_{r}^{d}(t)+\mathbf{G}_{v} \dot{\mathbf{q}}_{r}^{d}(t)+\mathbf{G}_{p} \mathbf{q}_{r}^{d}(t)
$$


so that the joint error $\mathbf{e}(t)=\mathbf{q}_{r}(t)-\mathbf{q}_{r}^{d}(t)$, satisfies,

$$
\ddot{\mathbf{e}}(t)+\mathbf{G}_{v} \dot{\mathbf{e}}(t)+\mathbf{G}_{p} \mathbf{e}(t)=\mathbf{0}
$$

The above model-based controller (equation(34)), thus results in the following set of closed-loop system of equations,

$$
\begin{aligned}
\ddot{\mathbf{q}}_{r}(t) & =\mathbf{u}_{I} \\
\mathbf{M}_{f f} \ddot{\mathbf{q}}_{f}+\mathbf{h}_{f}(\mathbf{q}, \dot{\mathbf{q}})+\mathbf{c}_{f}(\mathbf{q})+\mathbf{K} \mathbf{q}_{f} & =-\mathbf{M}_{r f}^{T} \mathbf{u}_{I}
\end{aligned}
$$

We note that, the response of the joint variables $\left(\mathbf{q}_{r}\right)$, would track the reference input $\left(\mathbf{q}_{r}^{d}\right)$ satisfactorily, for the proper choice of the gain matrices $\mathbf{G}_{p}$ and $\mathbf{G}_{v}$. The generalized flexible variables $\left(\mathbf{q}_{f}\right)$, on the other hand, although are coupled to control input $\mathbf{u}_{I}$ through the non-zero rows of the mass matrix $\mathbf{M}_{r f}^{T}$, would give bounded oscillatory response(Chedmail et al. 1991). These flexible vibrations however, can be actively damped by switching the control input to $\mathbf{u}_{I I}$ at the end of gross motion of links. The control input $\mathbf{u}_{I I}$ is computed by linearizing the closed-loop equations(38-39) around a point of the desired trajectory $\mathbf{q}^{d}$. We choose to linearize the system around the desired position, at the end of gross motion of links, that is, at $\mathbf{q}^{d}=\left(\mathbf{q}_{r}^{d^{T}}, \mathbf{q}_{f}^{d^{T}}=\mathbf{0}\right)^{T}$, where $\mathbf{q}_{r}^{d}$ is the desired joint position at the end of motion, and $\mathbf{0}$ is $1 \times N$ zero vector. We note that, desired velocity $\left(\dot{\mathbf{q}}^{d}\right)$ and acceleration $\left(\ddot{\mathbf{q}}^{d}\right)$ are zero at this position. The control input $\mathbf{u}_{I I}$ will then be,

$$
\mathbf{u}_{I I}=-\left[\mathbf{G}_{L Q R}\right] \delta \mathbf{x}
$$

where $\delta \mathbf{x}=\left(\mathbf{e}^{T}, \mathbf{q}_{f}^{T}, \dot{\mathrm{e}}^{T}, \dot{\mathbf{q}}_{f}^{T}\right)^{T}$ is the $2(n+N)$ full state vector: joint error and flexible variables. $\mathbf{G}_{L Q R}$ is the $n \times 2(n+N)$ optimal feedback gain matrix determined using the classical LQR method with a quadratic performance index in terms of the state and the input.

\section{Model Comparison}

Comparison of several dynamic modeling approaches for rigid-link manipulators is reported by Ju and Mansour(1989). However, no attempt has been made to compare different discretization models to develop the dynamics in case of flexible-link robot manipulators. In this section, we compare the assumed modes and finite element models.

A word is in order about the number of modes (or elements) to be considered in the discretization model formulation. Several researchers (Hastings and Book 1986, Singh and Schy 1986 a, Book 1990) have suggested to retain only the first few modes in the model. Justification for retaining only fewer modes in the model is based on the high frequency and low amplitudes of terms that are dropped and the fact that the actuators and sensors cannot operate in the high frequency range (Cannon and Schmitz 1984). Unfortunately, it is somewhat an art to figure out how many modes should be retained for complex systems 


\begin{tabular}{|c|c|c|c|c|}
\hline Mode & \multicolumn{3}{|c|}{ Number of Elements } & \multirow{2}{*}{$\begin{array}{c}\text { Exact } \\
\text { No. }\end{array}$} \\
\cline { 2 - 4 } & 1 & 2 & 3 & Values \\
\hline 1 & $2.0963 e+2$ & $2.0873 e+2$ & $2.0864 e+2$ & $2.0864 e+2$ \\
\hline 2 & $2.0654 e+3$ & $1.3186 e+3$ & $1.3118 e+3$ & $1.3075 e+3$ \\
\hline 3 & & $4.4597 e+3$ & $3.7067 e+3$ & $3.6611 e+3$ \\
\hline 4 & & $1.2944 e+4$ & $8.3473 e+3$ & $7.1742 e+3$ \\
\hline 5 & & & $1.5709 e+4$ & $1.1860 e+4$ \\
\hline 6 & & & $3.1318 e+4$ & $1.7716 e+4$ \\
\hline
\end{tabular}

Table 1: Natural frequencies $(H z)$ of a clamped-free beam

such as space structures, with many vibration modes of nearly the same frequency. In this case, the exact number of modes to be included, can be determined from modal cost analysis techniques (Skelton et al. 1982, Book 1990). For robot manipulators, on the other hand, a relatively small number of modes (two or three) suffices to represent the flexible dynamics (Tsujisawa and Book 1989). The higher modes, however, should be included in the model, if it is likely that these modes may excite the servo-loop frequencies. It should be noted that the first $m$ calculated vibratory modes can be considered within acceptable accuracy, when $m$-element model is used in the FEM model using minimal order polynomial interpolation functions (Przemieniecki 1968). For example, consider the natural frequencies (see table 1) of a clamped-free beam (with the system parameters of link 2 from table 3 ). We observe that first $m$-exact values of the natural frequencies of the beam (Meirovitch 1986) are within acceptable accuracy, when $m$-element model with cubic interpolation functions (equation(16)) is used for the clamped-free beam. Note also that the finite element model consistently over-estimates the exact values of the natural frequencies (see table 1).

\subsection{Ease of Modeling}

In both the assumed modes and finite element models, the flexible deformation is represented by a linear combination of spatial functions (also called admissible functions) multiplying time dependent mode amplitudes or nodal coordinates, respectively. In the assumed modes model, these admissible functions are global, in the sense that they are defined over the entire length of the beam, and they belong to the class of trigonometric functions. This leads to complicated integrations that are involved in the mass and stiffness matrix computations. Indeed, it is not feasible to get closed form solutions for manipulator links of complex geometry. The orthogonal assumptions of these functions however simplifies the number of terms in the mass and stiffness matrices and results in diagonal matrix structure. Finally, because the admissible functions are normally from a 'complete set' (Meirovitch 1986), convergence to the actual solution is guaranteed. 
In the finite element model, on the other hand, the admissible functions are local, in the sense that they are defined over small subdomains of the link, and they belong to the class of polynomial functions, that are simple and easy to work with. Because the admissible functions are local, it is easy to get closed form solutions for manipulator links with complex geometry. The admissible functions are all the same for every element and they are 'nearly' orthogonal. Indeed, it can be shown that the shape functions of an element $i$ will only contribute to the neighboring elements $i-1$ and $i+1$ and this in turn leads to banded mass and stiffness matrices. Since the local interpolation functions do not fall within the definition of a complete set, the monotonic convergence cannot always be guaranteed(Meirovitch 1986).

The finite element model formulation has an additional advantage in that, it can be extended to the closed-loop manipulators (Sunada and Dubowsky 1981), and to robot links of complex geometrical shapes (Jonker 1990) without much additional effort, due to the 'local' property of the admissible functions. We can also use 3-dimensional beam elements for efficient link design of the flexible robot arms (Meirovitch 1986, Jonker 1990). Moreover, symbolic computation of the equations of motion can be readily adapted to automation, as it merely consists of suitably assembling the element matrices (see appendix) for the manipulator system.

\subsection{Time-dependent Frequency Equation}

One of the complexity of the assumed modes model (see $\S 2.1 .1$ ) is that the frequency equation becomes time dependent when exact clamped-mass conditions are used for certain configurations of flexible manipulators. Although this fact was pointed out by De Luca and Siciliano(1991), and Tadikonda and Baruh(1992), they have neglected the time-dependent terms in the frequency equation for their model derivation. Neglecting the time-dependent terms may lead to inaccurate mode shapes and over-estimated eigen frequencies. In the sequel, we present a novel method to solve the time-dependent frequency equation.

Let us rewrite the clamped-mass frequency equation(12) as,

$$
\begin{array}{r}
f\left(\beta_{i}, M, J, D\right)=\left(1+\cosh \beta_{i} \cos \beta_{i}\right)-M \beta_{i}\left(\cosh \beta_{i} \sin \beta_{i}-\sinh \beta_{i} \cos \beta_{i}\right) \\
-J \beta_{i}{ }^{3}\left(\cosh \beta_{i} \sin \beta_{i}+\sinh \beta_{i} \cos \beta_{i}\right)+M J \beta_{i}{ }^{4}\left(1-\cosh \beta_{i} \cos \beta_{i}\right) \\
-D^{2} \beta_{i}{ }^{4}\left(1-\cosh \beta_{i} \cos \beta_{i}\right)-2 D \beta_{i}{ }^{2} \sinh \beta_{i} \sin \beta_{i}=0
\end{array}
$$

We note that when the terms $M, J$, and $D$ change continuously in time, the solutions $\left(\beta_{i}\right)$ of the frequency equation also change continuously in time. This is based on the property that the frequency equation is continuous in $\beta_{i}$. Since the roots of the frequency equation are all distinct, we can differentiate equation(41) with respect to time,

$$
\frac{d f\left(\beta_{i}, M, J, D\right)}{d t}=\frac{\partial f}{\partial \beta_{i}} \frac{d \beta_{i}}{d t}+\frac{\partial f}{\partial M} \frac{d M}{d t}+\frac{\partial f}{\partial J} \frac{d J}{d t}+\frac{\partial f}{\partial D} \frac{d D}{d t}=0
$$


and rearrange to obtain,

$$
\frac{d \beta_{i}}{d t}=\frac{-\left(\frac{\partial f}{\partial M} \frac{d M}{d t}+\frac{\partial f}{\partial J} \frac{d J}{d t}+\frac{\partial f}{\partial D} \frac{d D}{d t}\right)}{\left(\frac{\partial f}{\partial \beta_{i}}\right)}
$$

where

$$
\begin{aligned}
& \frac{\partial f}{\partial M}=\beta_{i}\left(\sinh \beta_{i} \cos \beta_{i}-\cosh \beta_{i} \sin \beta_{i}+\beta_{i}{ }^{3} J\left(1-\cosh \beta_{i} \cos \beta_{i}\right)\right) \\
& \frac{\partial f}{\partial J}=-\beta_{i}{ }^{3}\left(\sinh \beta_{i} \cos \beta_{i}+\cosh \beta_{i} \sin \beta_{i}-\beta_{i} M\left(1-\cosh \beta_{i} \cos \beta_{i}\right)\right) \\
& \frac{\partial f}{\partial D}=-2 \beta_{i}{ }^{2}\left(\sinh \beta_{i} \sin \beta_{i}+\beta_{i}{ }^{2} D\left(1-\cosh \beta_{i} \cos \beta_{i}\right)\right)
\end{aligned}
$$

and

$$
\begin{aligned}
\frac{\partial f}{\partial \beta_{i}}= & \sinh \beta_{i} \cos \beta_{i}\left(1+M-\beta_{i}{ }^{2}(2 D+3 J)+\beta_{i}{ }^{4}\left(D^{2}-M J\right)\right) \\
& -\cosh \beta_{i} \sin \beta_{i}\left(1+M+\beta_{i}{ }^{2}(2 D+3 J)+\beta_{i}{ }^{4}\left(D^{2}-M J\right)\right) \\
& -2 \beta_{i}{ }^{3} \cosh \beta_{i} \cos \beta_{i}\left(J-2\left(D^{2}-M J\right)\right) \\
& -2 \beta_{i} \sinh \beta_{i} \sin \beta_{i}(M+2 D)-4 \beta_{i}{ }^{3}\left(D^{2}-M J\right)
\end{aligned}
$$

It should be noted that the ordinary differential equation(43) on $\beta_{i}$, is also a function of the generalized position and velocity variables of the manipulator system, and hence it can be solved together with the equations of motion along with the initial condition $\beta_{i}(t=0)$. The initial value $\left(\beta_{i}(t=0)\right)$ can be computed from the frequency equation(41).

For the numerical simulation of a flexible RRP manipulator (see section 5 ), we have neglected the contributions of $M_{D L}$ and $J_{L}$ in clamped-mass boundary conditions for the prismatic jointed flexible link 3 (see figure 3). For this example the differential equation reduces to

$$
\frac{d \beta_{i}}{d t}=\frac{f_{1}\left(\beta_{i}, l_{3}\right)}{f_{2}\left(\beta_{i}, l_{3}\right)} \frac{d l_{3}}{d t}
$$

where

$$
\begin{aligned}
f_{1}\left(\beta_{i}, l_{3}\right)= & \frac{m_{p}}{\rho_{3} A_{3} l_{3}^{2}} \beta_{i}\left(\sinh \beta_{i} \cos \beta_{i}-\cosh \beta_{i} \sin \beta_{i}\right) \\
f_{2}\left(\beta_{i}, l_{3}\right)= & \left(1+\frac{m_{p}}{\rho_{3} A_{3} l_{3}}\right)\left(\sinh \beta_{i} \cos \beta_{i}-\cosh \beta_{i} \sin \beta_{i}\right) \\
& -\frac{2 m_{p}}{\rho_{3} A_{3} l_{3}} \beta_{i} \sinh \beta_{i} \sin \beta_{i}
\end{aligned}
$$

Note that $l_{3}(t)$, and $\frac{d l_{3}}{d t}$ are the generalized position and velocity variables for the prismatic jointed flexible link 3. 


\subsection{Over-estimation of Stiffness Matrix}

It is known in literature that use of finite element models to approximate the flexibility, usually gives rise to over estimation of structure stiffness matrix $(\mathbf{K})$ for the system model (Meirovitch 1986) (see also table 1). We show in the rest of this section, that over estimation of stiffness may lead to unstable closed-loop response of the original system, using the modelbased control law. Let us rewrite the control law, with the computed version of stiffness matrix $(\widehat{\mathbf{K}})$ as,

$$
\boldsymbol{\Gamma}=\left(\mathbf{M}_{r r}-\mathbf{M}_{r f} \mathbf{M}_{f f}^{-1} \mathbf{M}_{r f}^{T}\right) \mathbf{u}_{I}+\left(\mathbf{h}_{r}+\mathbf{c}_{r}-\mathbf{M}_{r f} \mathbf{M}_{f f}^{-1}\left(\mathbf{h}_{f}+\mathbf{c}_{f}+\widehat{\mathbf{K}} \mathbf{q}_{f}\right)\right)
$$

Then the closed-loop equations of motion of the manipulator system is given by,

$$
\begin{aligned}
\ddot{\mathbf{e}}(t)+\mathbf{G}_{v} \dot{\mathbf{e}}(t)+\mathbf{G}_{p} \mathbf{e}(t) & =-\mathbf{M}_{r r}^{-1} \mathbf{M}_{r f}\left(\mathbf{M}_{f f}-\mathbf{M}_{r f}^{T} \mathbf{M}_{r r}^{-1} \mathbf{M}_{r f}\right)^{-1} \Delta \mathbf{K} \mathbf{q}_{f}(5) \\
\mathbf{M}_{f f} \ddot{\mathbf{q}}_{f}+\mathbf{h}_{f}+\mathbf{c}_{f}+(\mathbf{K}-\mathbf{H} \Delta \mathbf{K}) \mathbf{q}_{f} & =-\mathbf{M}_{r f}^{T} \mathbf{u}_{I}
\end{aligned}
$$

where $\mathbf{H}=\mathbf{M}_{r f}^{T} \mathbf{M}_{r r}^{-1} \mathbf{M}_{r f}\left(\mathbf{M}_{f f}-\mathbf{M}_{r f}^{T} \mathbf{M}_{r r}^{-1} \mathbf{M}_{r f}\right)^{-1}, \Delta \mathbf{K}=(\widehat{\mathbf{K}}-\mathbf{K})$, and $\mathbf{K}$ is the original stiffness matrix of the manipulator system.

\section{Remarks:}

1. The matrix $\mathbf{M}_{f f}$ is symmetric, and positive definite. This follows from the definition of the system mass matrix $\mathbf{M}(\mathbf{q})$ (see appendix).

2. The matrix $\mathbf{H}$ is positive semi-definite.

PROOF. The matrix $\mathbf{M}_{r r}$ is symmetric, and positive definite from the definition of $\mathbf{M}(\mathbf{q})$, and the rank of $\mathbf{M}_{r f}$ is equal to that of $\mathbf{M}_{r r}$ and that is equal to $n$, where $n$ is the number of joints. Therefore, the congruence transformation $\mathbf{M}_{r f}^{T} \mathbf{M}_{r r}^{-1} \mathbf{M}_{r f}$ is symmetric, positive semi-definite and of rank $n$. By Schur's complement(Horn and Johnson 1985) of the system mass matrix $\mathbf{M}(\mathbf{q})$, the matrix $\left(\mathbf{M}_{f f}-\mathbf{M}_{r f}^{T} \mathbf{M}_{r r}^{-1} \mathbf{M}_{r f}\right)^{-1}$ is also symmetric, and positive definite, and of rank $N$, where $N$ is the number of flexible generalized variables. Note that usually $N \gg n$. Hence from the property of rank of product matrices, the rank of the matrix $\mathbf{H}$ is $n$ and therefore is positive semi-definite.

3. The necessary condition for the above closed-loop system of equations(52-53) to give stable response for $\mathbf{q}_{f}$, is that the mass matrix $\mathbf{M}_{f f}$ and the closed-loop stiffness matrix $(\mathbf{K}-\mathbf{H} \Delta \mathbf{K})$, are positive definite.

Lemma: In the model-based decoupling control law, if the flexural structure stiffness matrix is over-estimated (i.e. $\Delta \mathbf{K}>0$ ), the closed-loop of flexible manipulator system is stable only if $\left[\alpha \lambda_{\max }(\mathbf{H})\right]<1.0$, where $\alpha$ is the percentage error in over-estimation of the stiffness matrix.

PROOF. Let us define $\lambda_{\max }(\mathbf{K}) \equiv \lambda_{K}, \lambda_{\max }(\mathbf{H}) \equiv \lambda_{H}$, and $\lambda_{\max }(\Delta \mathbf{K}) \equiv \alpha \lambda_{K}$. Then condition for the matrix $(\mathbf{K}-\mathbf{H} \Delta \mathbf{K})$ to be positive definite reduces to a condition that 
$\alpha \lambda_{H}<1.0$. Hence if this condition holds, in the case of over-estimated stiffness matrix, the closed-loop system will be stable.

The above lemma clearly indicates that flexible manipulator system could be effectively controlled using the model-based control law only if proper care is taken in the finite element model formulation. In particular, the lemma can be used to determine the maximum allowable estimation error tolerance of the model stiffness parameters, so that the response of closed-loop of original manipulator system is stable.

\subsection{Computational Complexity}

Book(1984) first reported the number of mathematical operations(i.e. multiplications and additions) required for the inverse dynamics computation of revolute jointed flexible link manipulators. Li and Sankar(1993) later modified the algorithm and reported an improvement in computational efficiency for the inverse dynamics computations. It should be mentioned that Book(1984) addressed chiefly the problem of recursive dynamic computations and did not discuss issues of computations to derive the closed form equations. Moreover, the payload contribution to the computation counts is not addressed in both of their formulations. Li and Sankar(1993) presented the number of mathematical operations required for inertia matrix computations in terms of total number of links, the number of flexible links, and the number of assumed modes. However, further simplifications of terms can be obtained, if the geometric, inertial, and mode shapes are numerically specified before the symbolic calculations. The resulting form of equations, would be ideally suited for dynamic modelbased real-time controller implementations, since it would minimize the computational load, and also unlike the recursive forms, the inverse dynamics calculations for a particular joint torque (or force) can be computed independent of other joints (Cetinkunt and Ittoop 1992). It should be noted that no such computation counts is reported in the literature for the finite element based flexible link manipulators, and for flexible manipulators having prismatic joints.

We present the number of mathematical operations required for inertia matrix computation for the robot configurations of RR, RRR, RP, and RRP, due to assumed modes and finite elements. For the RR and RP configuration robots both the links are considered flexible, while for the RRR and RRP configuration robots, the first link is considered rigid and the last two links are considered flexible. Each flexible link is approximated by two assumed modes or two elements in the respective models. The computation counts are listed in Table 2. It should be noted that in the present study, the time independent parameters like geometric, inertial, and mode shapes are specified numerically before deriving symbolic expressions for the elements of the inertia matrix (see algorithm 1 of appendix).

The inertia matrix computation counts due to Li and Sankar(1993) are almost an order less compared to the present formulation, however their expression for the computation counts (see Table II on pp.89 of Li and Sankar(1993)), gives erroneous results in certain cases. For example, if we compute the number of mathematical operations required for 


\begin{tabular}{|c|c|c|c|c|c|c|c|c|}
\hline \multirow{3}{*}{$\begin{array}{c}\text { flexible } \\
\text { robot } \\
\text { configuration }\end{array}$} & \multirow{2}{*}{\multicolumn{2}{|c|}{$\frac{{ }^{\dagger} \operatorname{Book}(1984)}{\mathrm{AMM}^{1}}$}} & \multirow{2}{*}{\multicolumn{2}{|c|}{$\frac{{ }^{\ddagger} \mathrm{Li} \& \text { Sankar(1993) }}{\text { AMM }}$}} & \multicolumn{4}{|c|}{ †Present Study } \\
\hline & & & & & \multicolumn{2}{|c|}{$\mathrm{AMM}$} & \multicolumn{2}{|c|}{$\mathrm{FEM}^{2}$} \\
\hline & mult. & add. & mult. & add. & mult. & add. & mult. & add. \\
\hline $\mathrm{RR}$ & 3755 & 3665 & 44 & 49 & 405 & 168 & 156 & 80 \\
\hline RRR & 4922 & 4851 & 92 & 102 & 878 & 376 & 592 & 237 \\
\hline $\mathrm{RP}$ & - & - & - & - & 103 & 42 & 88 & 33 \\
\hline RRP & - & - & - & - & 743 & 284 & 376 & 128 \\
\hline
\end{tabular}

$\dagger$ Inverse Dynamics Computation Counts

$\ddagger$ Inertia Matrix Computation Counts
1 Assumed Modes Model

2 Finite Element Model

Table 2: Computation counts for flexible-arm dynamics

one-flexible link manipulator with one-mode, we get -48 multiplications and -48 additions. Li and Sankar(1993) also do not account for the payload contributions. The computation counts due to Book(1984) is presented for completeness, though it is for the inverse dynamics computations.

It can be observed from the table 2, that the number of mathematical operations required for inertia matrix computation are fewer in case where the flexibility is approximated by the finite elements as compared to the assumed modes model. This is mainly due to the transformation matrix that describe the link deformations. We note that in case of assumed modes model this transformation matrix is computed according to equation(8) or equation(9), where the contribution due to each mode is evaluated at the tip of the link and summed over all the modes to describe the orientation and position of the tip of a flexible link. In the case of finite element model, the position and orientation of the tip of a flexible link can be described simply by the element nodal displacements and rotations at the tip of the link (see equation(17) or equation(18)). The required number of mathematical operations become even higher when the payload is also considered in the assumed modes formulation. For example, to compute the inertia matrix of a RR configuration robot with a payload, when both the links are considered flexible, we require 909 multiplications and 366 additions in case of assumed modes formulation with two modes, while we require only 178 multiplications and 98 additions for the finite element model formulation when each flexible link is discretized by two elements.

In the finite element model of an $n$-link manipulator with $n_{f}(\leq n)$ flexible links each with $m$-elements, the total number of second order differential equations of motion will be $4 m n_{f}+n$ for the spatial case and $2 m n_{f}+n$ for the planar case. In the assumed modes model of an $n$-link manipulator with $n_{f}$ flexible links with $m$-modes for each flexible link, the number of second order differential equations of motion will be $2 m n_{f}+n$ in the spatial case and $m n_{f}+n$ in planar case. In addition, for the assumed modes model, we need to solve $2 n_{f}$ nonlinear transcendental equations in the spatial case and $n_{f}$ equations for the 
planar case. If we use the method outlined in $\S 4.2$, then we need to solve additional $2 m n_{f}$ first order differential equations for the spatial case and $m n_{f}$ equations for the planar case.

It can be observed that the finite element model has $2 m n_{f}\left(m n_{f}\right.$ for the planar case) more first order differential equations compared to an equivalent assumed modes model. Hence, inspite of the lesser number of multiplications and additions for the inertia matrix (see table 2) of the finite element model, the 'cpu run-time' for numerical simulation studies will be more for finite element models. This fact is seen in the numerical simulation of the spatial RRP configuration manipulator (see section 5).

It may be noted that for real-time model-based control of flexible manipulators the simulation time is of less importance than the number of multiplications and additions required for the model evaluation. In the case of finite element models the number of multiplications and additions is significantly lower as compared to the assumed modes models. Hence, the finite element models are more suitable for model-based real-time control implementations.

\section{$5 \quad$ Numerical Results}

In this section, we compare the dynamic response of a flexible, spatial, RRP configuration robot (see figure 3), due to discretization models viz., assumed modes and finite elements. The manipulator is assumed to operate in a gravity-free environment. The revolute jointed link 2, and the prismatic jointed link 3, are considered flexible in the numerical simulation. Both the links are approximated by two modes or two elements in the respective models. The numerical simulation was performed on an IRIS-4D Workstation. The first-order differential equations of motion (state-space form) with the model-based control input (see equation(34)) were solved by a variable step, variable order (of interpolation), predictor-corrector (PECE), Adams algorithm (Shampine and Gordon 1975). The desired joint trajectory was chosen to be a smooth sinusoidal profile with zero-velocity and zero-acceleration at the start and at the end of trajectory. The joint 1 is commanded to move from $0^{\circ}$ to $180^{\circ}$ in 0.5 seconds, while the joint 2 is commanded to move from $0^{\circ}$ to $90^{\circ}$ during the same time period. The prismatic jointed flexible link, on the other hand, is extended from $0.3 \mathrm{~m}$ to $1.0 \mathrm{~m}$ in 0.5 seconds. The desired joint trajectories are shown in figure 4. Table 3 lists the physical system parameters used for the simulation. The cpu time used for the numerical simulation in the case of assumed modes formulation is 1533.34 seconds, and 2036.89 seconds in the case of finite element formulation.

Figures 5 and 6 show the time history plot of joint and end-effector position errors for the model-based decoupling controller, in cases of assumed modes and finite element models. It can be seen, that during trajectory tracking phase (between 0 and $0.5 \mathrm{sec}$ ), though the joint errors are close to zero, the end-effector position errors are in the order of $8 \mathrm{~mm}$, due to the oscillatory response of flexible variables (see figures 7-8). These flexible vibrations are then damped by the optimal(LQR) second loop control law, at the end of gross joint motions, and hence the end-effector position errors are also driven to acceptable limits (within $0.1 \mathrm{~mm}$ ) 


\begin{tabular}{ll}
\hline Physical system parameters & Value \\
\hline mass of link 1 $\left(m_{1}\right)$ & $3.7051 \mathrm{~kg}$ \\
mass of link 2 $\left(m_{2}\right)$ & $0.3310 \mathrm{~kg}$ \\
mass of link $3\left(m_{3}\right)$ & $0.4303 \mathrm{~kg}$ \\
mass of payload $\left(m_{p}\right)$ & $0.0828 \mathrm{~kg}$ \\
length of link 1 $\left(l_{1}\right)$ & $0.1 \mathrm{~m}$ \\
length of link 2 $\left(l_{2}\right)$ & $1.0 \mathrm{~m}$ \\
total length of link $3\left(l_{3}^{0}\right)$ & $1.3 \mathrm{~m}$ \\
rotary inertia of joint $1\left(I_{1}\right)$ & $0.352 \mathrm{kgm}^{2}$ \\
rotary inertia of joint 2 $\left(I_{2}\right)$ & $3.2 \mathrm{kgm}^{2}$ \\
flexural rigidity of link $2(E I)$ & $1165.4916 \mathrm{Nm}^{2}$ \\
flexural rigidity of link $3(E I)$ & $1165.4916 \mathrm{Nm}^{2}$ \\
\hline
\end{tabular}

Table 3: RRP configuration robot system parameters

between 0.5 and 1 second.

Figures 7 and 8 show the time history plot of flexural deflections and rotations at the end of links 2 and 3, respectively. It can be observed that the dynamic response of the flexible variables is similar for both the discretization models, except that the response of flexible variables due to finite element discretization lags behind the corresponding flexible variable response of the assumed modes model. This is due to the fact that the finite element approximation generally over-estimates the natural frequencies of the original system and this can be seen from figures 9 and 10. Note that the natural frequencies of the flexible manipulator system are proportional to the closed-loop eigenvalues of the system. We computed the closed-loop eigenvalues of manipulator system using the jacobian of the closed-loop equations of motion in state-space form. It should be noted from figures 9 and 10 that the frequencies of the first 2 vibratory modes of assumed modes model are within acceptable accuracy, when 2-element model is used in FEM model and this is in confirmity with the observation made by Przemieniecki(1968). Another feature is that the natural frequencies calculated using finite element model is always higher than the natural frequencies calculated using assumed modes model and this is clearly evident in the high frequency range (see figures $9-10$ between 0 and $0.3 \mathrm{sec}$ ). This over-estimated natural frequencies of FEM model however, converges to that of the assumed modes model in the lower frequency range (see figures 9-10). The closed loop imaginary eigenvalues of the flexible variables also illustrate the time-invariant property of the frequencies correspond to revolute jointed flexible link 2 , and time varying nature of frequencies correspond to the prismatic jointed flexible link 3 (see figures 9-10).

This numerical simulation study thus demonstrates the results of the preceding sections. In the next section, we summarize the main contributions of this paper, and present 
some concluding remarks.

\section{Summary and Conclusion}

In this paper, we have presented a systematic dynamic modeling procedure based on homogeneous transformation matrices, for spatial multi-link flexible manipulators with both revolute and prismatic joints. The flexibility of the links is approximated by discretization models namely the assumed modes, and the finite elements, and their representation is compared. Lagrangian formalism has been employed to derive closed form equations of motion of the flexible manipulator system. Computer algebra is used to yield the most computationally efficient form of the equations of motion that are ideally suited for dynamic model-based real-time controller implementations. We showed that fewer number of mathematical operations are required for inertia matrix computation in case of finite element model compared to the assumed modes formulation, however the number of differential equations of motion is larger for the finite element model. We addressed the issue of time-dependent frequency equation of assumed modes model, and presented a novel method to solve the frequency equation. We have also showed that over-estimation of stiffness of the original manipulator system may lead to unstable closed-loop response using a model-based control algorithm.

In conclusion, we recommend the assumed modes model formulation for discretization

of manipulator links with uniform cross-sectional geometries, and for single-link flexible manipulators. It is also more suitable for numerical simulation purposes. On the other hand, the finite element model formulation is particularly recommended for manipulator links with complex cross-sectional geometries, and also for flexible multi-link manipulator systems. The finite element model also requires only fewer computations that lends itself ideally suited for dynamic model-based real-time controller implementations.

\section{Acknowledgement}

The authors would like to thank the referees for their valuable suggestions and comments.

\section{Appendix}

\section{Symbolic Equations of Motion}

In the present study, the closed form equations of motion for the flexible RRP configuration robot (see figure 3) were derived using the symbolic software MACSYMA(Annon 1987), employing the following algorithms.

The mass matrix due to the links are computed using the algorithm 1 .

Algorithm 1: 
S1: declare the types of all the variables and initialize.

S2: define the vector of generalized position variables: $\mathbf{q}^{T}=\left(q_{r_{1}}, \cdots, q_{r_{n}}, q_{f_{1}}, \cdots, q_{f_{N}}\right)$.

S3: input the geometric, inertial and other time-independent parameters.

S4: input the transformation matrices: $\mathbf{A}_{1}^{0}, \mathbf{E}_{1}^{0}, \cdots, \mathbf{A}_{n}^{n-1}, \mathbf{E}_{n}^{n-1}$ (equations(1-2)).

S5: compute the position vectors: $\mathbf{r}_{1}^{0}, \cdots, \mathbf{r}_{n}^{0}$ (equation $\left.(6)\right)$.

FOR $j=1$ to $n$ step 1 DO

FOR $i=1$ to $(n+N)$ step $1 \mathrm{DO}$

S6.1: compute $\delta_{i} \mathbf{r}_{j}^{0}=\left(\frac{\partial \mathbf{r}_{j}^{0}}{\partial q_{i}}\right)$

FOR $k=i$ to $(n+N)$ step 1 DO

S6.2: compute $\delta_{k} \mathbf{r}_{j}^{0}=\left(\frac{\partial \mathbf{r}_{j}^{0}}{\partial q_{k}}\right)$

S6.3: compute $M_{i, k}^{j}=\int_{0}^{l_{j}} \rho_{j} A_{j}\left(\delta_{i} \mathbf{r}_{j}^{0}\right)^{T}\left(\delta_{k} \mathbf{r}_{j}^{0}\right) d x$

S6.4: compute $M_{i, k}^{l i n k}=M_{i, k}^{l i n k}+M_{i, k}^{j}$

NEXT $k$.

NEXT $i$.

$\operatorname{NEXT} j$.

It should be noted that in case of finite element model, the mass matrix for each element of a link is computed first using algorithm 1 . We then obtain the mass matrix due to links by summing the element mass matrices over all the elements for all the links. The algorithm similar to the above can be used to determine the mass matrices due to joints, and the payload. Finally, the total mass matrix of the manipulator system is computed as:

Algorithm 2:

FOR $i=1$ to $(n+N)$ step 1 DO

FOR $k=i$ to $(n+N)$ step 1 DO

S1: compute $M_{i, k}=M_{i, k}^{\text {link }}+M_{i, k}^{\text {joint }}+M_{i, k}^{\text {payload }}$

NEXT $k$.

\section{NEXT $i$.}

Using the Lagrangian formulation of dynamics, the vectors of Coriolis, centrifugal terms $(\mathbf{h}(\mathbf{q}, \dot{\mathbf{q}}))$, and the gravitational terms $(\mathbf{c}(\mathbf{q}))$, are then determined using the following algorithm.

Algorithm 3: 
S1: define the generalized position and velocity variables: $\mathbf{q}^{T}=\left(q_{r_{1}}, \cdots, q_{r_{n}}, q_{f_{1}}, \cdots, q_{f_{N}}\right)$, and $\dot{\mathbf{q}}^{T}=\left(\dot{q}_{r_{1}}, \cdots, \dot{q}_{r_{n}}, \dot{q}_{f_{1}}, \cdots, \dot{q}_{f_{N}}\right)$.

FOR $i=1$ to $(n+N)$ step 1 DO

$$
\begin{aligned}
& \text { S2.1: compute } h_{i}=\sum_{j=1}^{n+N} \sum_{k=1}^{n+N}\left(\frac{\partial M_{i, j}}{\partial q_{k}}-\frac{1}{2} \frac{\partial M_{j, k}}{\partial q_{i}}\right) \dot{q}_{j} \dot{q}_{k} \\
& \text { S2.2: compute } g_{i}=\sum_{j=1}^{n} \int_{0}^{l_{j}} \rho_{j} A_{j} \mathbf{g}^{T} \frac{\partial \mathbf{r}_{j}^{0}}{\partial q_{i}} d x
\end{aligned}
$$

NEXT $i$.

\section{Inverse of a partitioned Mass matrix}

The mass matrix of the complete flexible manipulator system is given by

$$
\mathbf{M}(\mathbf{q})=\left(\begin{array}{ll}
\mathbf{M}_{r r} & \mathbf{M}_{r f} \\
\mathbf{M}_{r f}^{T} & \mathbf{M}_{f f}
\end{array}\right)
$$

From Lagrangian formulation of dynamics, $\mathbf{M}(\mathbf{q})$ is symmetric and positive definite. Hence its inverse $\mathbf{M}^{-1}(\mathbf{q})$ exists, and is also symmetric and positive definite. We can show then for $\mathbf{M}(\mathbf{q})$, the inverse $\mathbf{M}^{-1}(\mathbf{q})$ is given by either,

$$
\mathbf{M}^{-1}(\mathbf{q})=\left(\begin{array}{cc}
\left(\mathbf{M}_{r r}-\mathbf{M}_{r f} \mathbf{M}_{f f}^{-1} \mathbf{M}_{r f}^{T}\right)^{-1} & -\mathbf{M}_{r r}^{-1} \mathbf{M}_{r f}\left(\mathbf{M}_{f f}-\mathbf{M}_{r f}^{T} \mathbf{M}_{r r}^{-1} \mathbf{M}_{r f}\right)^{-1} \\
-\left(\mathbf{M}_{f f}-\mathbf{M}_{r f}^{T} \mathbf{M}_{r r}^{-1} \mathbf{M}_{r f}\right)^{-1} \mathbf{M}_{r f}^{T} \mathbf{M}_{r r}^{-1} & \left(\mathbf{M}_{f f}-\mathbf{M}_{r f}^{T} \mathbf{M}_{r r}^{-1} \mathbf{M}_{r f}\right)^{-1}
\end{array}\right)
$$

or

$$
\mathbf{M}^{-1}(\mathbf{q})=\left(\begin{array}{cc}
\left(\mathbf{M}_{r r}-\mathbf{M}_{r f} \mathbf{M}_{f f}^{-1} \mathbf{M}_{r f}^{T}\right)^{-1} & -\left(\mathbf{M}_{r r}-\mathbf{M}_{r f} \mathbf{M}_{f f}^{-1} \mathbf{M}_{r f}^{T}\right)^{-1} \mathbf{M}_{r f} \mathbf{M}_{f f}^{-1} \\
-\mathbf{M}_{f f}^{-1} \mathbf{M}_{r f}^{T}\left(\mathbf{M}_{r r}-\mathbf{M}_{r f} \mathbf{M}_{f f}^{-1} \mathbf{M}_{r f}^{T}\right)^{-1} & \left(\mathbf{M}_{f f}-\mathbf{M}_{r f}^{T} \mathbf{M}_{r r}^{-1} \mathbf{M}_{r f}\right)^{-1}
\end{array}\right)
$$

By the fact that any principal submatrix of a positive definite matrix is positive definite (Horn and Johnson 1985), and by the above formulae for $\mathbf{M}^{-1}(\mathbf{q})$, we have $\left(\mathbf{M}_{r r}-\mathbf{M}_{r f} \mathbf{M}_{f f}^{-1} \mathbf{M}_{r f}^{T}\right)^{-1}$, and $\left(\mathbf{M}_{r r}-\mathbf{M}_{r f} \mathbf{M}_{f f}^{-1} \mathbf{M}_{r f}^{T}\right)$ are symmetric and positive definite. Similarly, $\left(\mathbf{M}_{f f}-\mathbf{M}_{r f}^{T} \mathbf{M}_{r r}^{-1} \mathbf{M}_{r f}\right), \mathbf{M}_{r r}, \mathbf{M}_{f f}$ are also symmetric and positive definite.

\section{References}

Alberts,T.E., Xia,H., and Chen,Y. 1992. Dynamic Analysis to Evaluate Viscoelastic Passive Damping Augmentation for the Space Shuttle Remote Manipulator System, ASME J. Dynamic Systems, Meas. and Control, Vol. 114, pp.468-475.

Annon. 1987. MACSYMA User's Guide, Symbolics, Inc. 
Asada,H., and Slotine,J-J.E. 1986. Robot Analysis and Control, John Wiley \& Sons, Inc.

Asada,H., Ma,Z.-D., and Tokumaru,H. 1990. Inverse Dynamics of Flexible Robot Arms: Modeling and Computation for Trajectory Control, ASME J. Dynamic Systems, Meas. and Control, Vol. 112, pp.177-185.

Barbieri,E., and Özgüner,Ü. 1988. Unconstrained and Constrained Mode Expansion for a Flexible Slewing Link, ASME J. Dynamic Systems, Meas. and Control, Vol. 110, pp.416-421.

Baruh,H., and Tadikonda,S.S.K. 1989. Issues in the Dynamics and Control of Flexible Robot Manipulators, AIAA J. Guidance, Control and Dynamics, 12(5):659-671.

Bayo,E. and Saharian,M. 1986. Timoshenko Versus Bernoulli Beam Theories for the Control of Flexible Robots, Proc. IASTED Int. Symp. Applied Control and Identification, pp.178-182.

Bellezza,F., Lanari,L., and Ulivi,G. 1990. Exact Modeling of the Flexible Slewing Link, Proc. IEEE Int. Conf. Robotics and Automation, pp.734-739.

Book,W.J. 1984. Recursive Lagrangian Dynamics of Flexible Manipulator Arms, Int. J. Robotics Res. 3(3):87-101.

Book,W.J. 1990. Modeling, Design, and Control of Flexible Manipulator Arms: A Tutorial Review, Proc. IEEE Conf. Decision and Control, pp.500-506.

Cannon,Jr.R.H., and Schmitz,E. 1984. Initial Experiments on the End-point Control of a Flexible One-link Robot, Int. J. Robotics Res. 3(3):62-75.

Cetinkunt,S., and Book,W.J. 1987. Symbolic Modeling of Flexible Manipulators, Proc. IEEE Int. Conf. Robotics and Automation, pp.2074-2080.

Cetinkunt,S., and Ittoop,B. 1992. Computer-Automated Symbolic Modeling of Dynamics of Robotic Manipulators with Flexible Links, IEEE Trans. Robotics and Automation, RA-8(2):94-105.

Chalhoub,N.G., and Ulsoy,A.G. 1986. Dynamic Simulation of a Lead-Screw Driven Flexible Robot Arm and Controller, ASME J. Dynamic Systems, Meas. and Control, Vol. 108, pp.119-126.

Chedmail,P., Aoustin,Y., and Chevallereau,Ch. 1991. Modelling and Control of Flexible Robots, Int. J. Numerical Methods in Engineering, Vol. 32, pp.1595-1619.

Chiang, Wen-Wei, Kraft, R., and Cannon,Jr.R.H. 1991. Design and Experimental Demonstration of Rapid, Precise End-Point Control of a Wrist Carried by a Very Flexible Manipulator, Int. J. Robotics Res. 10(1):30-40. 
Das,A., and Singh,S.N. 1990. Dual Mode Control of an Elastic Robotic Arm: Nonlinear Inversion and Stabilization by Pole Assignment, Int. J. Systems Science, 21(7):11851204.

De Luca,A., and Siciliano,B. 1991. Closed-Form Dynamic Model of Planar Multilink Lightweight Robots, IEEE Trans. Systems, Man and Cybernetics, SMC-21(4):826839.

De Luca,A., and Siciliano,B. 1993. Inversion-based Nonlinear Control of Robot Arms with Flexible Links, AIAA J. Guidance, Control and Dynamics, 16(6):1169-1176.

Fresonke,D.A., Hernandez,E., and Tesar,D. 1988. Deflection Prediction for Serial Manipulators, Proc. IEEE Int. Conf. Robotics and Automation, pp.482-487.

Good,M.C., Sweet,L.M., and Strobel,K.L. 1985. Dynamic Models for Control System Design of Integrated Robots and Drive Systems, ASME J. Dynamic Systems, Meas. and Control, Vol. 107, pp.53-59.

Hastings,G.G., and Book,W.J. 1986. Verification of a Linear Dynamic Model for Flexible Robotic Manipulators, Proc. IEEE Int. Conf. Robotics and Automation, pp.1024-1029.

Horn,R.A., and Johnson,C.R. 1985. Matrix Analysis, Cambridge University Press.

Huston,R.L. 1980. Flexibility Effects in Multibody System Dynamics, Mechanics Research Communications, Vol. 7, pp.261-268.

Jonker,J.B. 1990. A finite element dynamic analysis of flexible manipulators, Int. J. Robotics Res. 9(4):59-74.

Ju,M.S., and Mansour,J.M. 1989. Comparison of Methods for Developing the Dynamics of Rigid-body Systems, Int. J. Robotics Res. 8(6):19-27.

Korolov,V.V., and Chen,Y.H. 1989. Controller Design Robust to Frequency Variation in a One-Link Flexible Robot Arm, ASME J. Dynamic Systems, Meas. and Control, Vol. 111, pp.9-14.

Li,C.-J., and Sankar,T.S. 1993. Systematic Methods for Efficient Modeling and Dynamics Computation of Flexible Robot Manipulators, IEEE Trans. Systems, Man and Cybernetics, SMC-23(1):77-94.

Low,K.H. 1989. Solution Schemes for the System Equations of Flexible Robots, J. Robotic Systems, 6(4):383-405.

Meirovitch,L. 1986. Elements of Vibration Analysis, 2nd ed., McGraw-Hill Book Co. 
Morris,K.A., and Vidyasagar,M. 1990. A Comparison of Different Models for Beam Vibrations From the Standpoint of Control Design, ASME J. Dynamic Systems, Meas. and Control, Vol. 112, pp.349-356.

Naganathan,G., and Soni,A.H. 1987. Coupling Effects of Kinematics and Flexibility in Manipulators, Int. J. Robotics Res. 6(1):75-84.

Oakley,C.M, and Cannon,Jr.R.H. 1988. Initial Experiments on the Control of a Two-link Manipulator with a Very Flexible Forearm, Proc. American Control Conf., pp.996-1002.

Oakley,C.M, and Cannon,Jr.R.H. 1989. End-point Control of a Two-link manipulator with a Very Flexible Forearm: Issues and Experiments, Proc. American Control Conf., pp.1381-1388.

Przemieniecki,J.S. 1968. Theory of Matrix Structure Analysis, McGraw-Hill Book Co., pp.328-339.

Rayna,G. 1987. REDUCE Software for Algebraic Computation, Springer-Verlag.

Rovner,D.M., and Cannon,Jr.R.H. 1987. Experiments Toward On-Line Identification and Control of a Very Flexible One-Link Manipulator, Int. J. Robotics Res. 6(4):3-19.

Sakawa,Y., and Luo,Z.H. 1989. Modeling and Control of Coupled Bending and Torsional Vibrations of Flexible Beams, IEEE Trans. Automatic Control, AC-34(9):970-977.

Shampine,L.F., and Gordon,M.K. 1975. Computer Solution of Ordinary Differential Equations: The Initial Value Problem, W.H. Freeman \& Co.

Shiffman,J.J. 1993. Lyapunov Functions and the Control of the Euler-Bernoulli Beam, Int. J. Control, 57(4):971-990.

Siciliano,B., and Book,W.J., 1988. A Singular Perturbation Approach to Control of Light weight Flexible Manipulators, Int. J. Robotics Res. 7(4):79-90.

Singh,S.N., and Schy,A.A. 1986 a. Control of Elastic Robotic Systems by Nonlinear Inversion and Modal Damping, ASME J. Dynamic Systems, Meas. and Control, Vol. 108, pp.180-189.

Singh,S.N., and Schy,A.A. 1986 b. Elastic Robot Control: Nonlinear Inversion and Linear Stabilization, IEEE Trans. Aerospace and Electronic Systems, AES-22(4):340-347.

Skelton,R.E., Hughes,P.C., and Hablani,H.B. 1982. Order Reduction for Models of Space Structures Using Modal Cost Analysis, AIAA J. Guidance, Control and Dynamics, 5(4):351-357. 
Sunada,W., and Dubowsky,S. 1981. The Application of Finite element methods to the Dynamic Analysis of Spatial and Coplanar Linkages, ASME J. Mechanical Design, Vol. 103, pp.643-651.

Tadikonda,S.S.K., and Baruh,H. 1992. Dynamics and Control of a Translating Flexible Beam with a Prismatic Joint, ASME J. Dynamic Systems, Meas. and Control, Vol. 114, pp.422-427.

Timoshenko,S., Young,D.H., and Weaver,Jr.W. 1974. Vibration Problems in Engineering, 4th ed., John Wiley \& Sons, Inc.

Tosunoglu,S., Lin,S.-H., and Tesar,D. 1992. Accessibility and Controllability of Flexible Robotic Manipulators, ASME J. Dynamic Systems, Meas. and Control, Vol. 114, pp.50-58.

Tsujisawa,T., and Book,W.J. 1989. A Reduced Order Model Derivation for Lightweight Arms with a Parallel Mechanism, Proc. IEEE Int. Conf. Robotics and Automation, pp.728-735.

Usoro,P.B., Nadira,R., and Mahil,S.S. 1986. A Finite Element/Lagrangian Approach to Modeling Lightweight Flexible Manipulators, ASME J. Dynamic Systems, Meas. and Control, Vol. 108, pp.198-205.

Wang,D., and Vidyasagar,M. 1989. Transfer Functions for a Single Flexible Link, Proc. IEEE Int. Conf. Robotics and Automation, pp.1042-1047.

Yang,G.-B., and Donath,M. 1988. Dynamic Model of a one-link Robot Manipulator with both Structural and Joint Flexibility, Proc. IEEE Int. Conf. Robotics and Automation, pp.476-481.

Yuh,J., and Young,T. 1991. Dynamic Modeling of an Axially Moving Beam in Rotation: Simulation and Experiment, ASME J. Dynamic Systems, Meas. and Control, Vol.113, pp.34-40. 


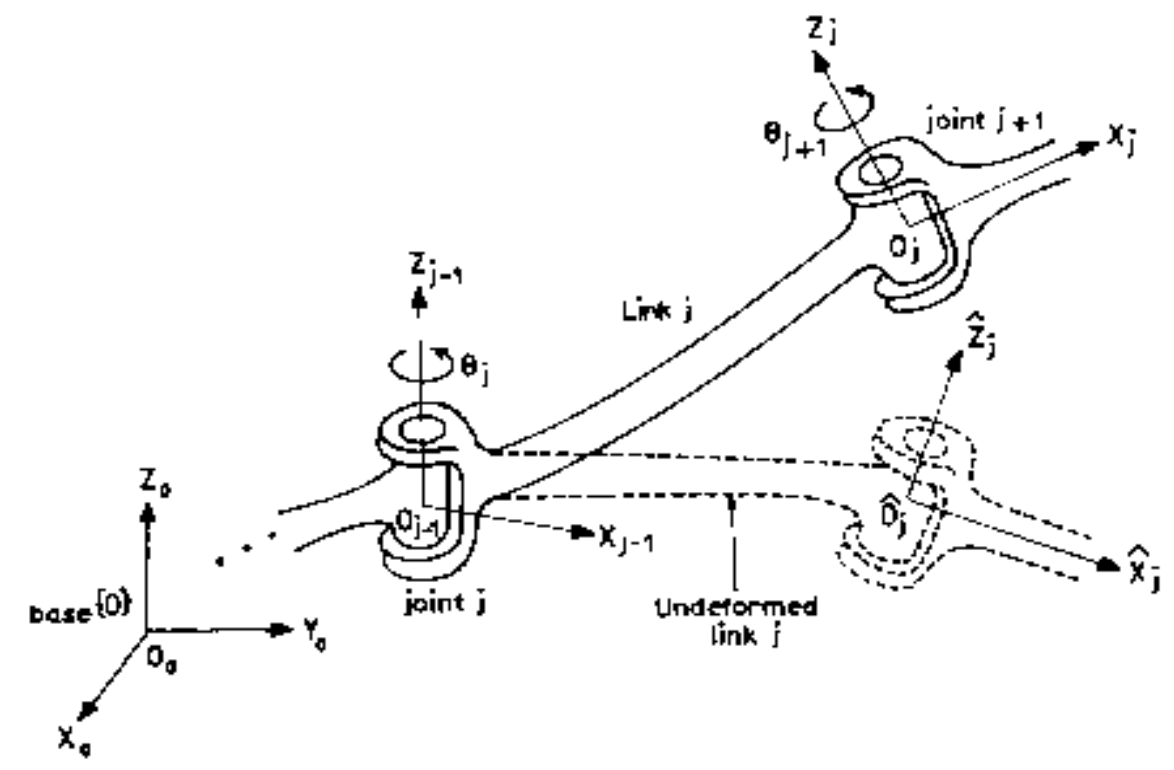

Figure 1: Coordinate systems for the flexible link $j$ 


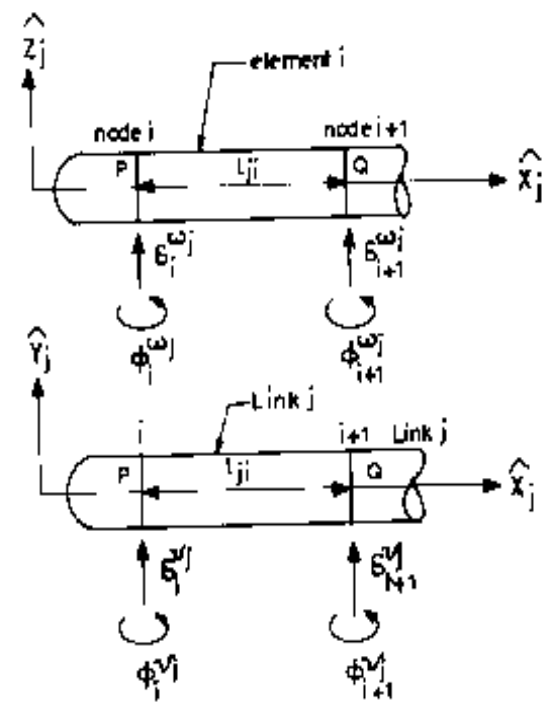

Figure 2: A beam element with nodal displacements in link $j$

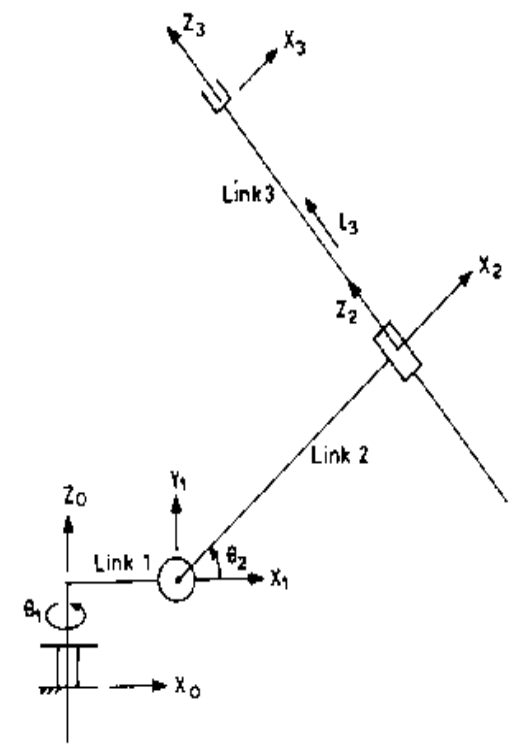

Figure 3: Flexible spherical(RRP) manipulator 

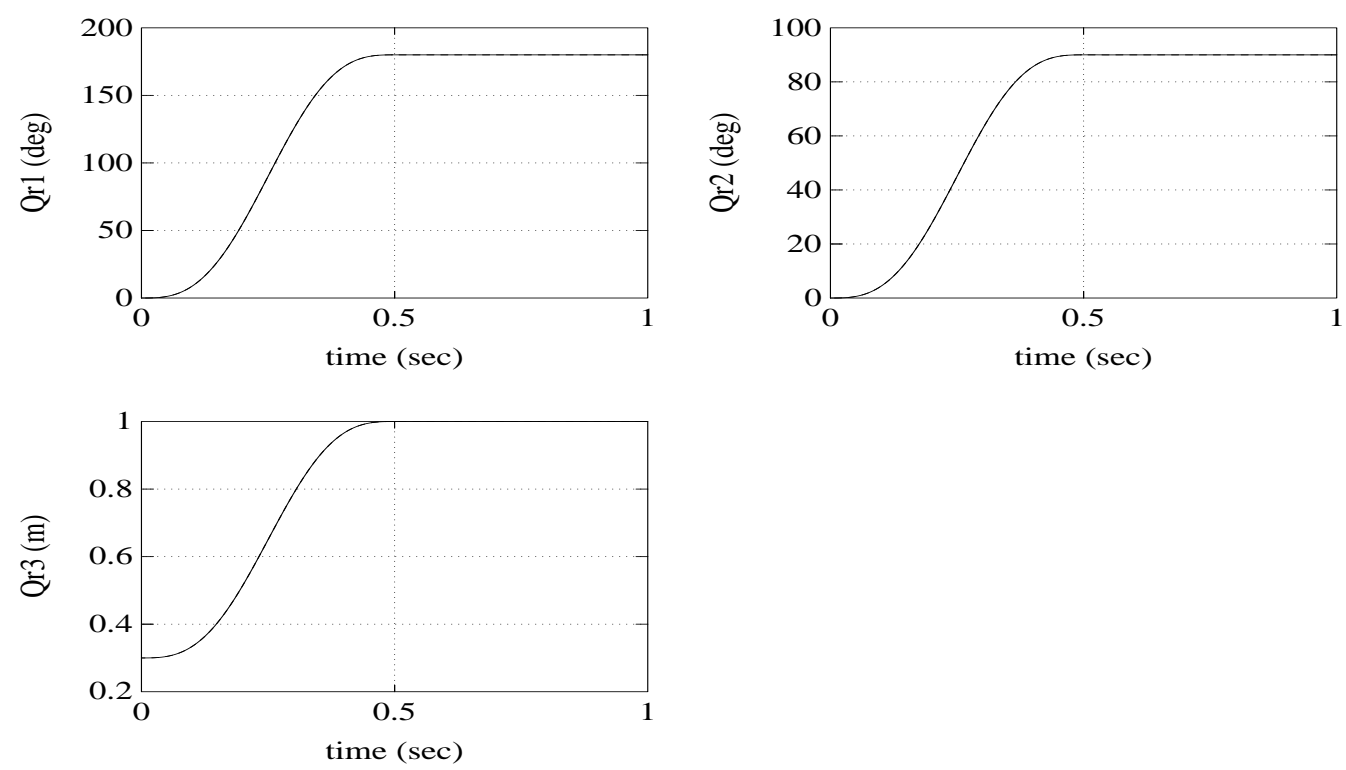

Figure 4: Desired and actual joint position trajectory
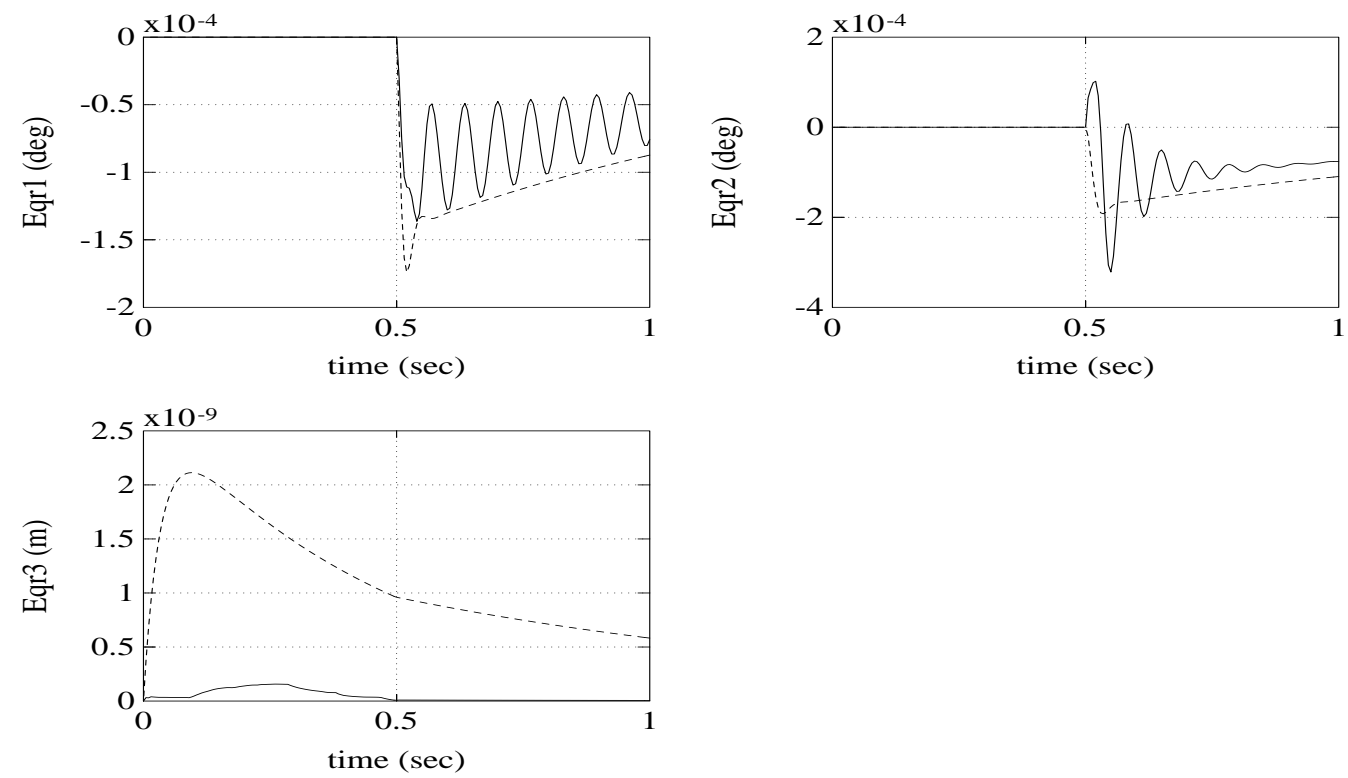

Figure 5: Time history of the joint position errors. (- : assumed modes model, - - - : finite element model) 

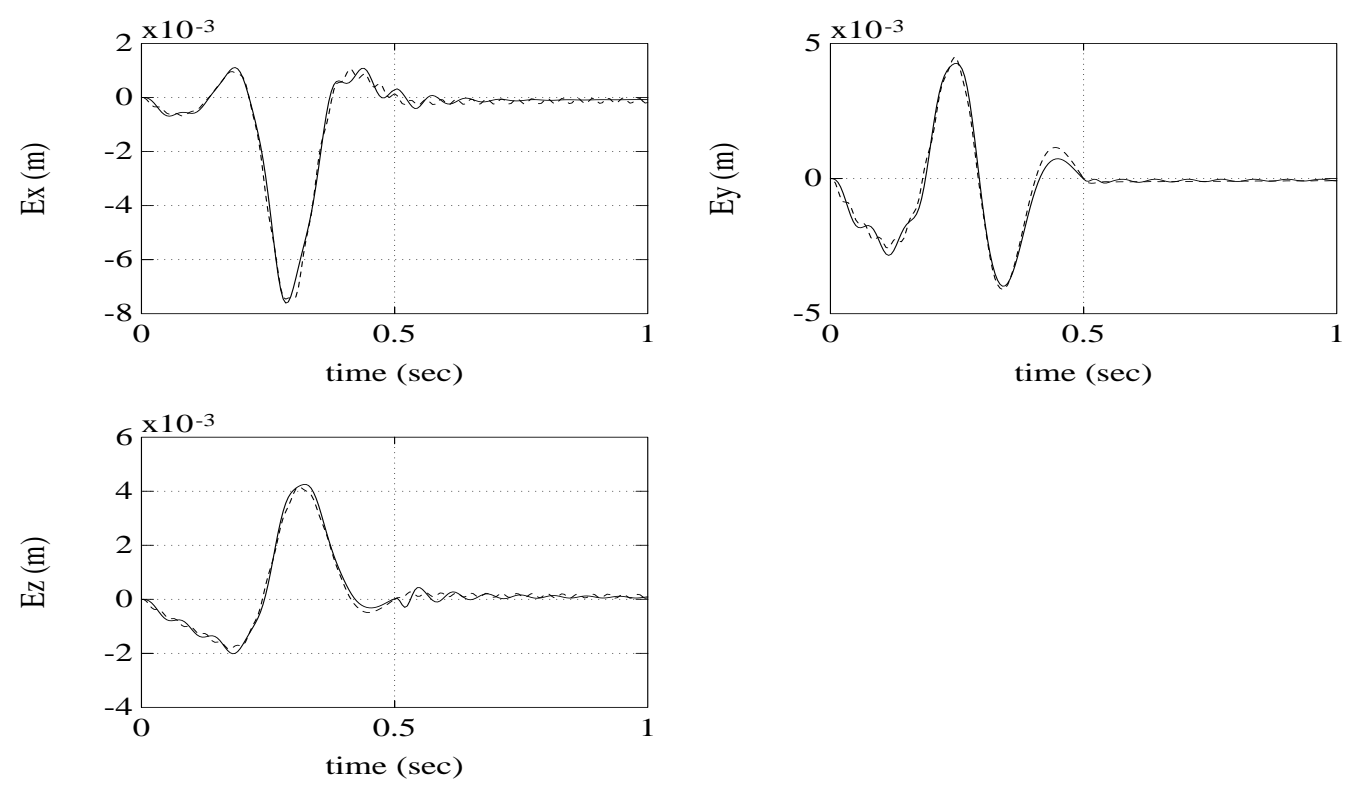

Figure 6: Time history of the end-effector position errors. ( - : assumed modes model, - - - : finite element model)
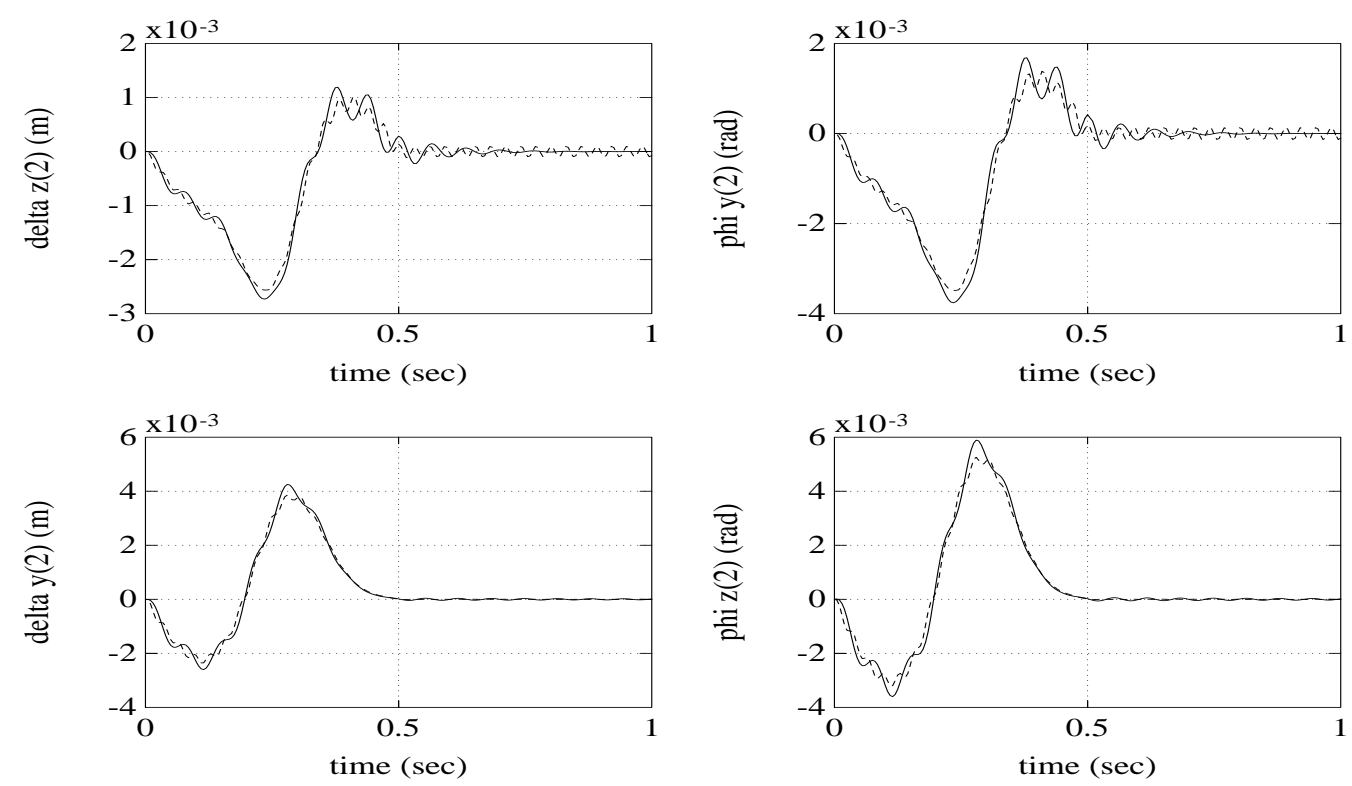

Figure 7: Time history of flexible deflection and rotation of the in-plane and out-of-plane components, at the tip of link 2. (— : assumed modes model, - - - : finite element model) 

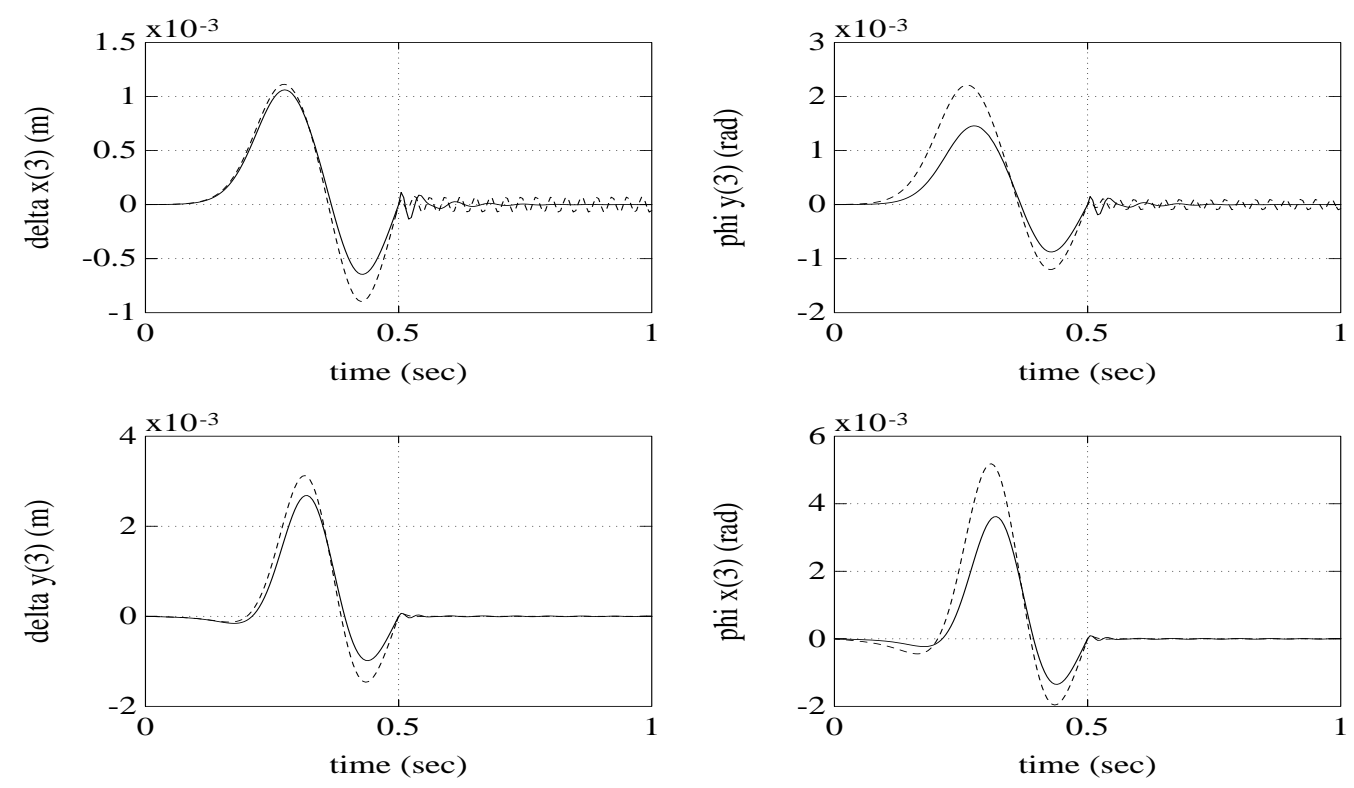

Figure 8: Time history of flexible deflection and rotation of the in-plane and out-of-plane components, at the tip of link 3. (- : assumed modes model, - - - : finite element model)

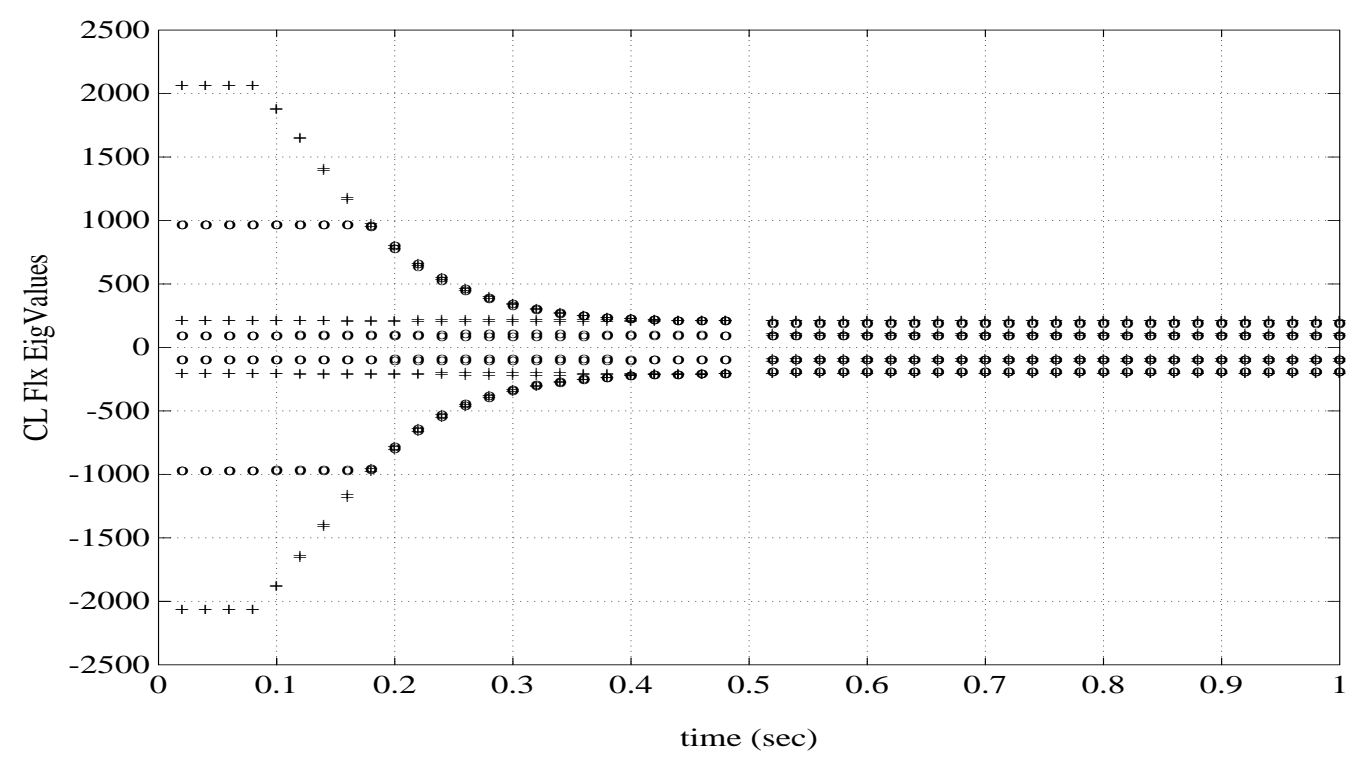

Figure 9: Time history of mode 1 closed-loop imaginary eigenvalues of flexible variables corresponding to links 2 and 3 . ( o : assumed modes model, + : finite element model ) 


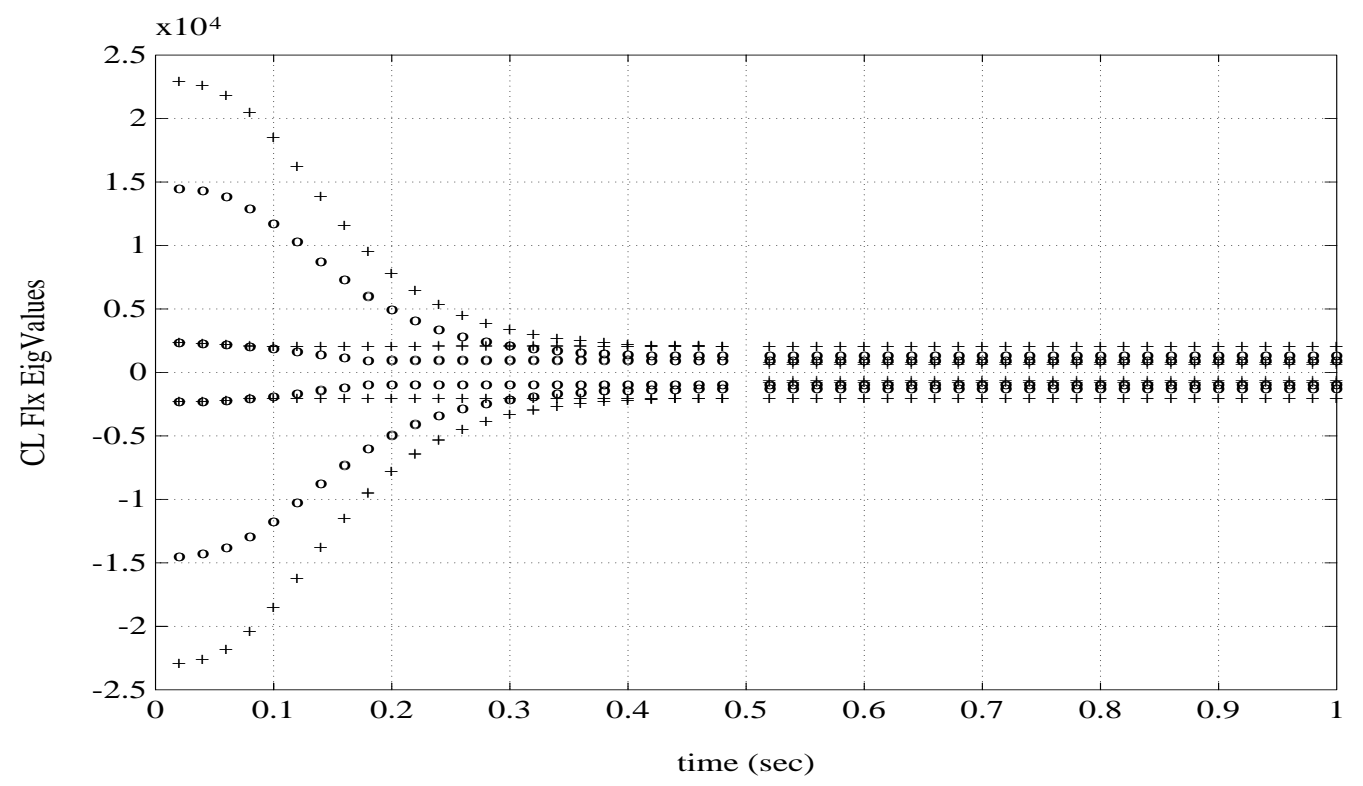

Figure 10: Time history of mode 2 closed-loop imaginary eigenvalues of flexible variables corresponding to links 2 and 3. ( o : assumed modes model, + : finite element model ) 